\title{
The Herschel Bright Sources (HerBS): sample definition and SCUBA-2 observations
}

\author{
Tom J. L. C. Bakx, ${ }^{1 \star}$ S. A. Eales, ${ }^{1}$ M. Negrello, ${ }^{1}$ M. W. L. Smith, ${ }^{1}$ E. Valiante, ${ }^{1}$ \\ W. S. Holland, ${ }^{2}$ M. Baes, ${ }^{3}$ N. Bourne, ${ }^{4}$ D. L. Clements, ${ }^{5}$ H. Dannerbauer, ${ }^{6,7,8}$ \\ G. De Zotti, ${ }^{9}$ L. Dunne, ${ }^{1}$ S. Dye,${ }^{10}$ C. Furlanetto, ${ }^{10,11}$ R. J. Ivison, ${ }^{4,12}$ S. Maddox, ${ }^{1}$ \\ L. Marchetti, ${ }^{13}$ M. J. Michałowski, ${ }^{4,14}$ A. Omont, ${ }^{15,16}$ I. Oteo, ${ }^{4,12}$ J. L. Wardlow, ${ }^{17}$ \\ P. van der Werf ${ }^{18}$ and C. Yang ${ }^{15,16,19,20,21}$
}

Affiliations are listed at the end of the paper

Accepted 2017 August 29. Received 2017 August 16; in original form 2017 January 23

\begin{abstract}
We present the Herschel Bright Sources (HerBS) sample, a sample of bright, high-redshift Herschel sources detected in the $616.4 \mathrm{deg}^{2}$ Herschel Astrophysical Terahertz Large Area Survey. The HerBS sample contains 209 galaxies, selected with a $500 \mu \mathrm{m}$ flux density greater than $80 \mathrm{mJy}$ and an estimated redshift greater than 2 . The sample consists of a combination of hyperluminous infrared galaxies and lensed ultraluminous infrared galaxies during the epoch of peak cosmic star formation. In this paper, we present Submillimetre CommonUser Bolometer Array 2 (SCUBA-2) observations at $850 \mu \mathrm{m}$ of 189 galaxies of the HerBS sample, 152 of these sources were detected. We fit a spectral template to the HerschelSpectral and Photometric Imaging Receiver (SPIRE) and $850 \mu \mathrm{m}$ SCUBA-2 flux densities of 22 sources with spectroscopically determined redshifts, using a two-component modified blackbody spectrum as a template. We find a cold- and hot-dust temperature of $21.29_{-1.66}^{+1.35}$ and $45.80_{-3.48}^{+2.88} \mathrm{~K}$, a cold-to-hot dust mass ratio of $26.62_{-6.74}^{+5.61}$ and a $\beta$ of $1.83_{-0.28}^{+0.14}$. The poor quality of the fit suggests that the sample of galaxies is too diverse to be explained by our simple model. Comparison of our sample to a galaxy evolution model indicates that the fraction of lenses are high. Out of the 152 SCUBA- 2 detected galaxies, the model predicts $128.4 \pm 2.1$ of those galaxies to be lensed ( 84.5 per cent). The SPIRE $500 \mu \mathrm{m}$ flux suggests that out of all 209 HerBS sources, we expect $158.1 \pm 1.7$ lensed sources, giving a total lensing fraction of 76 per cent.
\end{abstract}

Key words: gravitational lensing: strong-galaxies: high-redshift-submillimetre: galaxies.

\section{INTRODUCTION}

The Herschel Space Observatory (Pilbratt et al. 2010) has increased the number of known submillimetre galaxies (SMGs) from hundreds to hundreds of thousands. The Herschel Astrophysical Terahertz Large Area Survey (H-ATLAS; Eales et al. 2010; Valiante et al. 2016) is one of the largest legacies of Herschel. This survey observed a total of $616.4 \mathrm{deg}^{2}$ over five fields in five wavebands. The large-area surveys done with Herschel allow us to select sources that are among the brightest in the sky, of which a large percentage are lensed ultraluminous infrared galaxies (ULIRGs; $\left.10^{12} \mathrm{~L}_{\odot}<L_{\mathrm{FIR}}<10^{13} \mathrm{~L}_{\odot}\right)$ and hyperluminous infrared galaxies (HyLIRGs; $L_{\mathrm{FIR}}>10^{13} \mathrm{~L}_{\odot}$ ) at high redshift.
A similar selection for bright sources was already exploited in the $14.4 \mathrm{deg}^{2}$ Science Demonstration Phase (SDP) of H-ATLAS by Negrello et al. (2010), who used a simple flux cut-off to select lensed sources. They were able to remove all contaminants from their selection, local galaxies and blazars, and identified five lensed galaxies. Wardlow et al. (2013) followed a similar approach on the $94 \mathrm{deg}^{2}$ Herschel Multi-tiered Extragalactic Survey (HerMES) maps, and selected 13 sources with $S_{500 \mu \mathrm{m}}>100 \mathrm{mJy}$. Nine of these sources had follow-up data, done with the Submillimeter Array (SMA), the Hubble Space Telescope (HST), Jansky Very Large Array (JVLA), Keck and Spitzer. Wardlow et al. (2013) combined these data for six sources and confirmed their lensing nature, while three other sources had their lensing nature already confirmed by Borys et al. (2006), Conley et al. (2011) and Ikarashi et al. (2011). Recently, Negrello et al. (2017) and Nayyeri et al. (2016) used the same $S_{500 \mu \mathrm{m}}>100 \mathrm{mJy}$ flux density cut-off on the full 
H-ATLAS (616.4 $\mathrm{deg}^{2}$ ) and HerMES Large Mode Survey (HeLMS; $372 \mathrm{deg}^{2}$ ) maps, and created samples containing 77 and 80 sources, respectively. Spectroscopic and optical follow-up observations were able, so far, to confirm that 20 sources are indeed lensed, one is a protocluster (Ivison et al. 2013), while the remaining sources in Negrello et al. (2017) await more observations to be carried out to confirm their nature.

Large samples of lensed sources are interesting, both because of the lensed source and the intervening lensing galaxy (Treu 2010). The lensed source has an amplified flux density and increased angular size. The amplification in flux density allows us to study sources that would otherwise be too faint to detect. The increase in angular size allows us to study the internal properties of high-redshift sources with high resolution submm/mm and radio observatories, such as Atacama Large Millimeter/submillimeter Array (ALMA) and the Very Large Array (VLA). As most intervening, lensing sources are passively evolving ellipticals, they are submm dim and their contribution to the total measured flux density is minimal. This allowed ALMA Partnership (2015), Dye et al. (2015), Hatsukade et al. (2015), Rybak et al. (2015), Swinbank et al. (2015) and Tamura et al. (2015) to study SDP.81 down to sub-kiloparsec scales, using the increase in angular size in order to resolve the morphological and dynamical properties of a galaxy at a redshift of 3 .

Submm detected lensed sources, similar to SDP.81, are forming stars at rates of hundreds to several thousands of solar masses per year, and large samples of them can allow statistically significant studies into these extremely star-forming sources. This is important, because the comoving density of ULIRGs at $z=2-4$ is about a thousand times greater than in the local Universe, and these dusty star-forming galaxies are estimated to contribute about 10 per cent of the total star formation in this redshift range (Hughes et al. 1998; Blain et al. 1999; Smail et al. 2002; Wardlow et al. 2011; Casey, Narayanan \& Cooray 2014). This means that SMGs contribute significantly to the peak in cosmic star formation, which occurred around $z \sim 2.3$ (Chapman et al. 2005).

While the star formation rate of the Universe has been measured up to redshift $z \sim 8$ in rest-frame ultraviolet (UV) surveys, these studies only measure the unobscured star formation rates (Madau \& Dickinson 2014). The star formation processes in these dusty star-forming galaxies (DSFGs) tend to be obscured by the dust, and are missed by current optical investigations of the cosmic star formation rate. An added benefit of using submm observations to measure the obscured star formation rate is that submm flux density falls only slowly with redshift, because of the negative $K$-correction: submm observations observe the Rayleigh-Jeans part of the modified blackbody spectrum, which causes the flux density to increase as the galaxy's redshift increases. This increase is able to compensate for the cosmological dimming due to the increase of luminosity distance, e.g. a redshift 1 or 4 galaxy has a similar flux density in submm wavelengths (Blain \& Longair 1993; Blain et al. 2002; Bethermin et al. 2015).

The foreground galaxy's total mass (dark and baryonic) distribution determines the lensed morphology of the submm detected system (Vegetti et al. 2012; Hezaveh et al. 2016a,b). Therefore, highresolution imaging of the lensed morphology allows the detection of low-mass substructures in lensing galaxies. These substructures can then be used to test the formation of structure in large-scale cosmological simulations, such as the Millennium (Springel et al. 2005) and the recent EAGLE simulation (Schaye et al. 2015).

The statistics of galaxy-galaxy lensing systems furthermore allows for a measurement of global cosmological parameters. For example, the lensing statistics of 28 lensed quasars in the Sloan Digital
Sky Survey (SDSS) Quasar Lens Search (SQLS) gave an estimate of $\Omega_{\Lambda}=0.74 \pm 0.17$, assuming a spatially flat Universe (Oguri et al. 2012). Selecting lensed sources from bright submm samples is simple and unbiased method because it is based on the source, as the lens is usually faint in the submm. Eales (2015) showed that observations of a sample of 100 lensed Herschel sources would be enough to estimate $\Omega_{\Lambda}$ with a precision of 5 percent and observations of 1000 lenses would be enough to estimate $\Omega_{\Lambda}$ with a precision similar to that obtained from the Planck observations of the cosmic microwave background.

A high flux density cut-off ( $\left.S_{500 \mu \mathrm{m}}>100 \mathrm{mJy}\right)$ eliminates a large amount of possible lenses in order to achieve a low contamination rate from unlensed sources (González-Nuevo et al. 2012). Lowering the cut-off flux density to $80 \mathrm{mJy}$ was already tested in Wardlow et al. (2013). Out of the four galaxies with lensing verification, only one was confirmed to be a lens. In this paper, we will reinvestigate the question of using a lower cut-off flux, by selecting galaxies from the $616.4 \mathrm{deg}^{2} \mathrm{H}$-ATLAS survey. In order to decrease the contamination rate, we impose a photometric cut-off redshift $z_{\text {phot }}>2$ based on the Herschel-Spectral and Photometric Imaging Receiver (SPIRE) fluxes. The probability of lensing below this redshift falls off sharply, because of the smaller volume available between us and the source (Strandet et al. 2016). We will calculate the expected amount of lensed galaxies in our sample, by comparing the fluxes of our sources to a cosmological evolution model that takes lensing into account.

Our sample selection is based on Herschel fluxes, and a known problem of sources selected at $500 \mu \mathrm{m}$ with Herschel is the large solid angle of the beam (Scudder et al. 2016). This could lead to several sources blending into a single source, and result in a flux that is too large. This is why we observed the majority of our sources at $850 \mu \mathrm{m}$ with the Submillimetre Common-User Bolometer Array 2 (SCUBA-2) instrument on the James Clerk Maxwell Telescope (JCMT), whose beam has a six times smaller solid angle on the sky. The extra data point should also improve the photometric redshift estimates of our sources.

In Section 2, we discuss the selection of the Herschel Bright Sources (HerBS) sample, as well as the observations with SCUBA-2. We describe the results of the JCMT observations in Section 3, where we also remove several blazar contaminants from the sample. We rederive a spectral template for our sources with spectroscopically determined redshifts in Section 4. We discuss the effects of source confusion, the properties of the template, the redshift distribution of our sample and estimates of the lensing fraction in Section 5.

Throughout this paper we assume the $\Lambda$ cold dark model $(\Lambda \mathrm{CDM})$ model, and the best-fitting parameters found by the Planck Collaboration XIII (2016): $H_{0}=67.7 \mathrm{~km} \mathrm{~s}^{-1} \mathrm{Mpc}^{-1}$ and $\Omega_{\mathrm{M}}=$ 0.307 .

\section{SAMPLE AND MEASUREMENTS}

\subsection{The selection of the HerBS sample}

The sample was selected from the brightest, high-redshift sources in the H-ATLAS survey. The H-ATLAS survey used the Photodetector Array Camera and Spectrometer (PACS; Poglitsch et al. 2010) and SPIRE (Griffin et al. 2010) instruments on the Herschel Space Observatory to observe the North Galactic Pole (NGP) and South Galactic Pole (SGP) fields and three equatorial fields to a $1 \sigma$ sensitivity of $5.2 \mathrm{mJy}$ at $250 \mu \mathrm{m}$ to $6.8 \mathrm{mJy}$ at $500 \mu \mathrm{m}$, although the noise varies per source (Valiante et al. 2016). The three equatorial 
Table 1. The H-ATLAS fields.

\begin{tabular}{|c|c|c|c|c|c|c|c|}
\hline \multirow[t]{2}{*}{ Field } & \multicolumn{2}{|c|}{ Centre } & \multicolumn{2}{|c|}{ Approximate dimensions } & \multirow{2}{*}{$\begin{array}{l}\text { Final surface area } \\
\qquad\left(\mathrm{deg}^{2}\right)\end{array}$} & \multirow[t]{2}{*}{ Sources } & \multirow{2}{*}{$\begin{array}{l}\text { Surface density } \\
\quad\left(\mathrm{deg}^{-2}\right)\end{array}$} \\
\hline & RA $\left({ }^{h}: \mathrm{m} . \mathrm{s}\right)$ & Dec. $\left({ }^{\circ}:^{\prime}:{ }^{\prime \prime}\right)$ & $\mathrm{RA}\left({ }^{\circ}\right)$ & Dec. $\left({ }^{\circ}\right)$ & & & \\
\hline NGP & $13: 18: 00$ & 29:00:00 & 15 & 10 & 170.1 & 49 & 0.288 \\
\hline GAMA total & - & - & - & - & 161.6 & 72 & 0.446 \\
\hline GAMA 9 & 09:00:00 & 00:00:00 & 12 & 3 & 53.43 & 23 & 0.430 \\
\hline GAMA 12 & 12:00:00 & 00:00:00 & 12 & 3 & 53.56 & 26 & 0.485 \\
\hline GAMA 15 & 14:30:00 & 00:00:00 & 12 & 3 & 54.56 & 23 & 0.422 \\
\hline SGP & $23: 24: 46$ & $-33: 00: 00$ & 42 & 6 & 284.8 & 88 & 0.309 \\
\hline Total fields & - & - & - & - & 616.4 & 209 & 0.339 \\
\hline
\end{tabular}

Note. Reading from the left, the columns are: column 1 - name of field; columns 2 and 3 - the location of the centre of the field; columns 4 and 5 the approximate dimensions of the field; column 6 - the surface area from the final maps (Valiante et al. 2016); column 7 - the number of final HerBS sources in each field; column 8 - the surface density of HerBS sources per field.

fields overlap with the Galaxy And Mass Assembly (GAMA) fields 9,12 and $15 \mathrm{~h}$, and from here on we adopt this naming convention for the equatorial fields (Driver et al. 2011; Liske et al. 2015). The fields are defined in Table 1. In total the H-ATLAS survey detected approximately half a million sources.

We initially selected the HerBS sample from the H-ATLAS point source catalogues of Valiante et al. (2016), who extracted the flux densities at the $250 \mu \mathrm{m}$ position, and used this position for flux extraction at 350 and $500 \mu \mathrm{m}$. The flux densities in the catalogues are not deboosted, however the flux boosting is negligible compared to the flux uncertainty; around 1 per cent at $80 \mathrm{mJy}$, and diminishing for increasing flux density, as can be seen in table 6 of Valiante et al. (2016). We estimated the redshift of each source by fitting a source template to the 250, 350 and $500 \mu \mathrm{m}$ flux densities (Pearson et al. 2013). We selected the sources at an estimated redshift, $z_{\text {phot }}$, greater than 2 and a $500 \mu \mathrm{m}$ flux density, $S_{500 \mu \mathrm{m}}$, greater than $80 \mathrm{mJy}$. The source template was a two-temperature modified blackbody from Pearson et al. (2013) (see equation 3 and Table 5 in our Section 4). This modified blackbody was derived from the Herschel PACS and SPIRE flux densities of 40 sources with spectroscopically determined redshifts, with 25 sources at low redshifts $(z<1)$, and only 12 sources at high redshifts $(z>2)$. Our initial sample consisted of the 223 sources.

Where possible we removed sources that are coincident with a large nearby galaxy or a blazar (Negrello et al. 2010; López-Caniego et al. 2013). However, the pre-selection of blazars was not complete, and it only became clear after we had carried out the SCUBA2 observations that we had actually observed several blazars (see Section 3). The final HerBS sample consists of 209 submillimetre galaxies after removing all nearby galaxies and blazars, and is listed in Table A1. We plot the positions of the final 209 HerBS sources in the various fields in Fig. 1.

Several of the HerBS sources have been investigated individually. Fu et al. (2012) showed that HATLAS J114637.9-001132 (HerBS-2) is a strongly lensed submm galaxy, with a magnification between 7 and 17. Cox et al. (2011) and Bussmann et al. (2012) found that HATLAS J142413.9+022303 (HerBS-13) is a lensed submm galaxy, with a magnification of 4 . At a redshift of 4.24, the source has one of the highest redshifts in our sample. HATLAS J090311.6+003907 (HerBS-19) is also known as SDP.81, and has recently been observed by ALMA Partnership (2015). Negrello et al. (2010) showed SDP.81 is lensed using $880 \mu \mathrm{m}$ Submillimeter Array observations. Dye et al. (2015) and Tamura et al. (2015) reconstructed the galaxy from the ALMA observation, by modelling the distorting effect of the lens. They found a magnification of $\sim 11$.
This reconstructed image features details on the scale of hundreds of parsecs, and the image shows resolved individual giant molecular clouds in a $z=3.04$ galaxy. SDP.81 appears, through reconstructed HST and ALMA imaging, to be two interacting objects, where the dust disc is in a state of collapse.

However, not all our sources are lensed. Ivison et al. (2013) studied HATLAS J084933.4+021442 (HerBS-8), and found it was not a strongly lensed galaxy. Instead, it consists of multiple large galaxies in the process of merging, which has probably triggered starbursts in the individual galaxies, explaining the brightness in submm wavelengths.

Our HerBS sample overlaps partially with the sample from $\mathrm{Ne}$ grello et al. (2017), as 53 out of the 80 sources in their sample are also found in the HerBS sample. Their sample was designed specifically to find lensed systems, by imposing a flux density cut-off of $100 \mathrm{mJy}$ at $500 \mu \mathrm{m}$ and did not have a lower redshift limit.

\subsection{Observations with SCUBA-2}

We observed 203 sources with the SCUBA-2 array on the JCMT. The instrument consists of 10000 transition edge sensor (TES) bolometers, distributed over four arrays that observe at $450 \mu \mathrm{m}$ and four arrays that observe at $850 \mu \mathrm{m}$ (Holland et al. 2013). Both wavelengths are observed simultaneously, with the use of a dichroic mirror. The voltage across each array is optimized to ensure as many functional bolometers as possible. The optimized voltage places the majority of the bolometers within their sensitive resistance transition, whereupon any temperature fluctuation causes a current change. The resulting magnetic field variations are read out with separate Superconducting Quantum Interference Devices (SQUIDs) located under each bolometer.

The instrument scans the sky in a daisy pattern, circling around the source following a continuous petal-like track, providing a central 3 arcmin region of uniform exposure time, and keeping one part of the array on-source at all times (Chapin et al. 2013).

The observations conditions were in the grade- 3 weather band $\left[0.08<\tau_{1.3 \mathrm{~mm}}<0.12\right]$, which is only suitable for $850 \mu \mathrm{m}$ observations. The data were flux-calibrated against Uranus, Mars, CRL 618 and CRL 2688 (the Westbrook and Egg nebulae). The calibrators were observed between two and four times per observing run, and the flux calibration factors (FCFs) were estimated linearly for observations in between calibrators, and the closest calibrator was used otherwise (Dempsey et al. 2013).

Our observations consisted of $10 \mathrm{~min}$ exposures for each source. The bolometers are sampled at roughly $200 \mathrm{~Hz}$, and the data are 


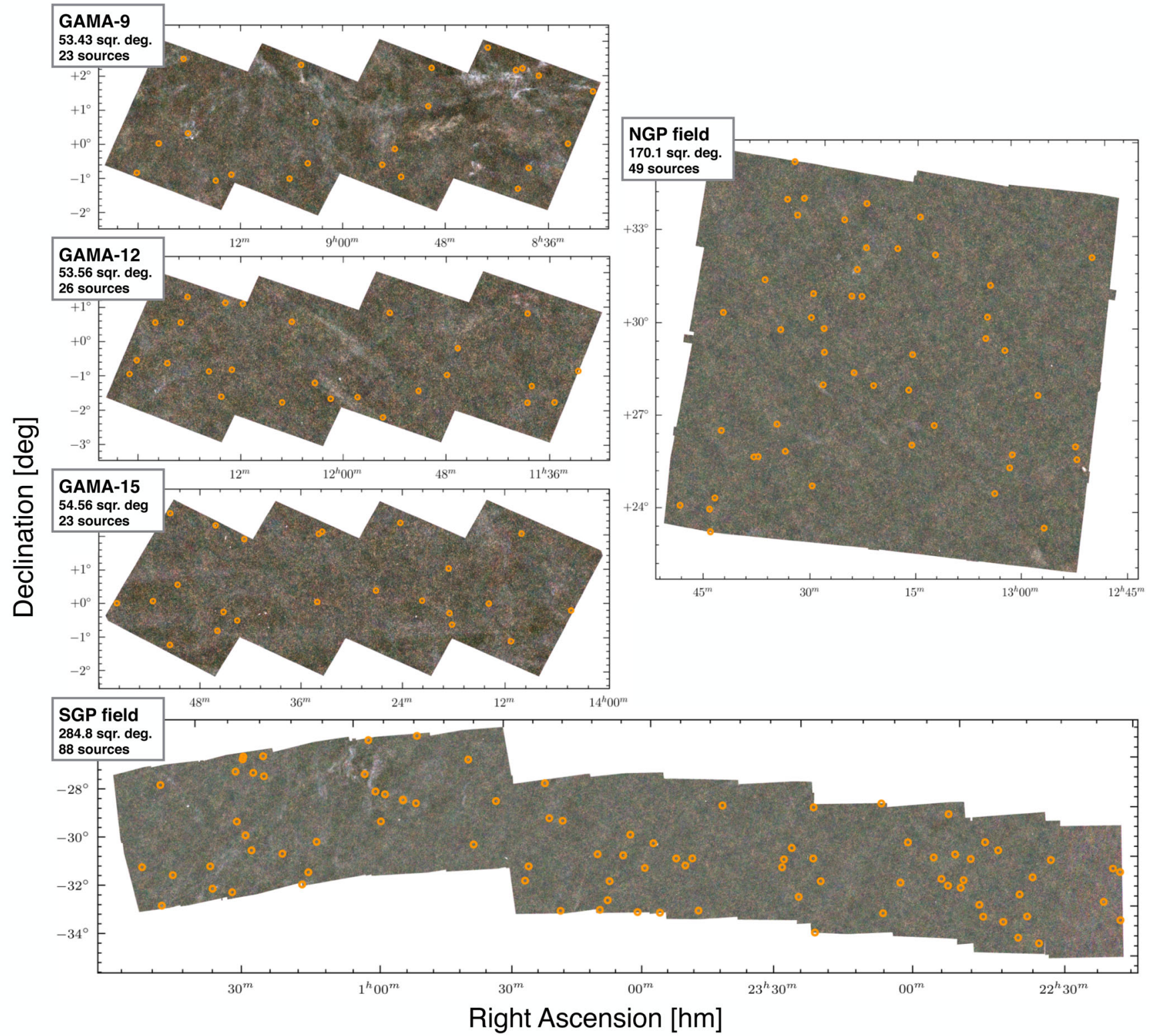

Figure 1. Herschel/SPIRE colour maps of the H-ATLAS fields. The orange circles mark the positions of the 209 HerBS sources. This figure is similar to fig. 2 in (Negrello et al. 2017), and shows how the sources are distributed over the sky.

stored in $30 \mathrm{~s}$ time slices for each of the arrays, where the first and last time slice of each exposure are flat-fields. Flat-fields probe the responsivity of individual bolometers, and are derived from the bolometer's response to the resistance heaters, which are located next to each bolometer.

\subsection{Data reduction}

The entire data reduction method is shown schematically in Fig. 2, and is described below. The data reduction was done with the ORAC_DR pipeline, which uses the KAPPA and SMURF packages from STARLINK, and the PICARD procedures (Chapin et al. 2010).

The basic data consist of the time-dependent signals from each bolometer and information about the specific scanning pattern of the arrays on the sky. The first step of the data reduction method flatfields and downsamples the data, to correct for individual bolometer performance and to reduce the file size by matching the sampling speed to the spatial scale of the maps. The second step removes the noise components in the signal iteratively, starting with the largest noise component (Chapin et al. 2013). Our final reduced map is achieved with additional data reduction steps: jackknife, fake point source injection and matched filtering. The final result is a $4 \times 4 \operatorname{arcmin}^{2}$ image with 1 arcsec resolution.

\subsubsection{The iterative data reduction step (make map)}

Sky emission is the dominant noise component, and it is shared by all bolometers. This common-mode signal (COM) is calculated by averaging the signals of all bolometers into one signal per subarray. The COM is then subtracted from the signal for each bolometer, taking care to adjust for individual bolometer amplification 


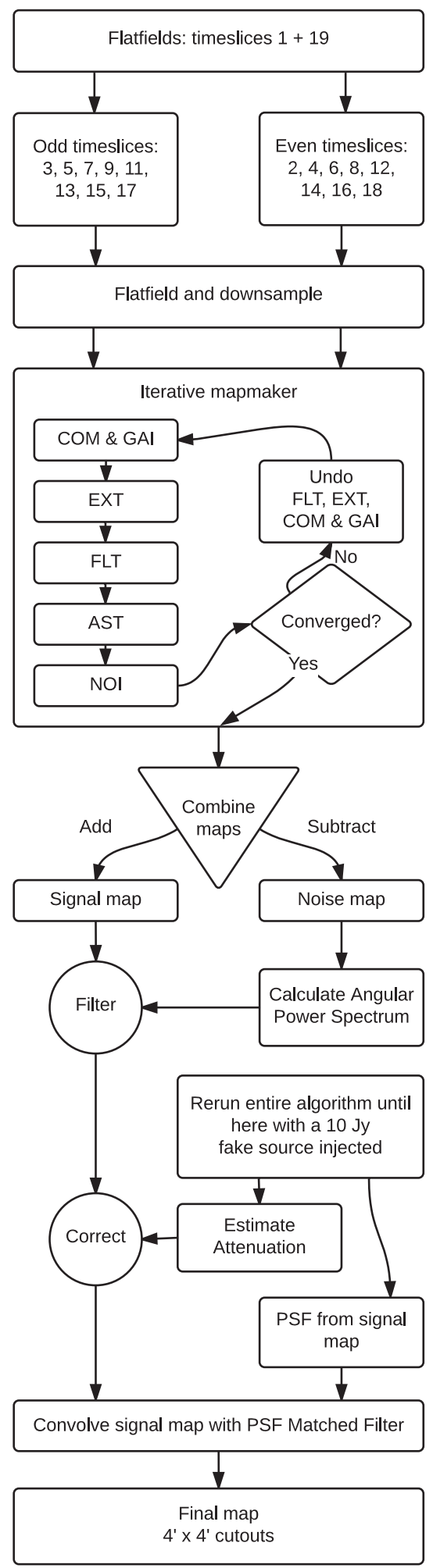

Figure 2. This flowchart shows the data reduction steps schematically, starting from the raw data files at the top, working to the reduced cutouts at the bottom. The intricacies are detailed in the data reduction section. For each observation, two sets of time slices are cleaned and processed through the iterative mapmaker, and these resulting maps are subtracted to provide a jackknife estimate of the noise. A fake source is injected to estimate peak attenuation due to the filtering process, and allows us to create a PSF for the final matched filter step. differences (GAI). Bolometers that have a signal that is inconsistent with the COM are rejected at this stage.

The signal is then corrected for the atmospheric extinction (EXT), a function of precipitable water vapour and telescope pitch, after which a high-pass Fourier filter (FLT) removes low frequency, $1 / f$ noise. The frequency cut-off is $0.8 \mathrm{~Hz}$, which corresponds to a spatial scale of 200 arcsec.

The next step removes the astronomical signal (AST) from the total signal, in order to estimate convergence of our iterative data reduction step. The signals of all bolometers are projected on to the sky, creating an astronomical map of our observation. Many data points contribute to the estimate of the astronomical signal in each spatial pixel, which greatly reduces the noise compared to the time series data. The map still contains noise, but the assumption made in this step of the iterative data reduction procedure is that everything in this map is real. The astronomical, space-domain map is then used to create a time-domain signal for each bolometer, by simulating an observation of our astronomical map. This is then removed from the signal for each bolometer.

The time-domain signal for each bolometer should now consist only of noise. This noise is calculated and compared to the convergence criterion (NOI), which is a minimum number of loops (four in this case) and a threshold noise level. If convergence is not reached in the NOI step, all the data processing steps (FLT, EXT, GAI and COM) are undone, except for the removal of the astronomical signal. This adds back the common-mode noise and the noise removed in the Fourier-filtering step. All the steps (see upper half of Fig. 2) are then repeated until the convergence criterion is met. After each cycle the new estimate of the astronomical signal is added to the previous estimate. The final image is obtained when the convergence criterion is met.

\subsubsection{Extra data reduction steps}

Apart from this standard data reduction procedure, shown in the top half of Fig. 2, we added the following additional steps.

For each source, we split the time slices into two sets. Each set consists of the flat-fields (first and last time slice) and either the odd or even half of the time slices. We ran the iterative mapmaker over each set separately, which allows us to execute a jackknife step (ORAC_DR procedure: SCUBA2_JACKKNIFE).

We used the iterative data reduction step to create a separate map for each half of the data. We subtracted one map from the other to create a noise map, from which we calculate the angular power spectrum of the noise. We used this angular power spectrum to construct a map-specific Fourier filter. A combined signal map is calculated by adding the two signal maps, and we then applied this Fourier filter to the signal map.

The high-pass filtering step attenuates the signal, and to account for this, we reran the entire data reduction algorithm with an injected fake source. This fake $10 \mathrm{Jy}$ point source [full width at halfmaximum (FWHM) of 13 arcsec - the main beam size of $850 \mu \mathrm{m}$ observations with JCMT; Dempsey et al. 2013] was injected into both the odd and even time slices, offset at 30 arcsec from the centre. This extremely bright, fake source allowed us to calculate an effective point spread function (PSF) and also provided an estimate of the signal attenuation due to the high-pass filtering, which usually was around 15-20 per cent.

Finally, we applied a matched filter to the signal map, in which we convolved our signal map with the PSF found by injecting a fake source. This provided the final, reduced observation map. We 
Table 2. SCUBA-2 observations of the HerBS sample.

\begin{tabular}{lcc}
\hline & Sources & Percentage \\
\hline HerBS galaxies & $\mathbf{2 0 9}$ & $\mathbf{1 0 0}$ \\
SCUBA-2 observed & $\mathbf{1 8 9}$ & $\mathbf{9 0 . 4}$ \\
Detected $\quad(>3 \sigma, \theta<10 \operatorname{arcsec})$ & 152 & 69.4 \\
Not detected $\quad(<3 \sigma)$ & 27 & 12.9 \\
Not detected $\quad(>3 \sigma, \theta>10 \operatorname{arcsec})$ & 10 & 8.1 \\
Not observed & $\mathbf{2 0}$ & $\mathbf{9 . 6}$ \\
Blazar contaminants & 14 & \\
\hline
\end{tabular}

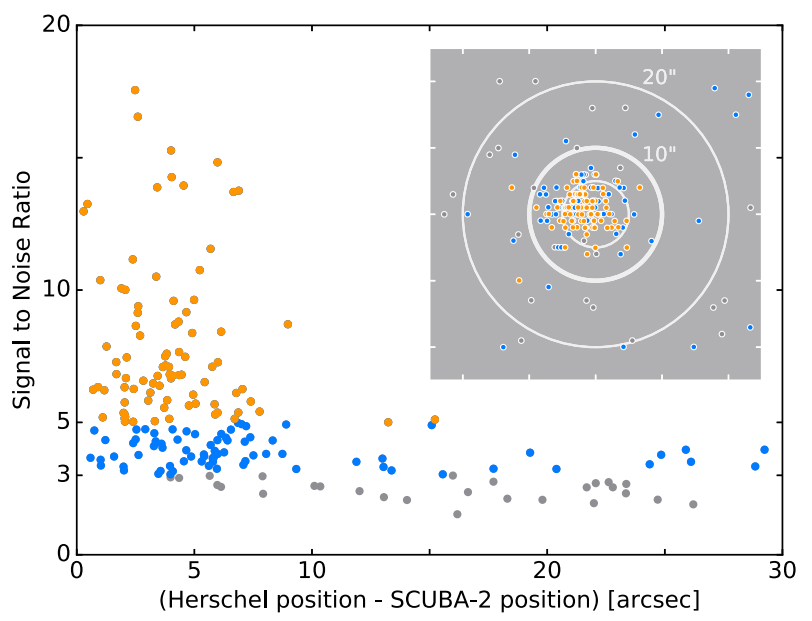

Figure 3. The majority of high signal-to-noise ratio SCUBA-2 fluxes lie in a 10 arcsec circle around the SPIRE position. We choose a cut-off signalto-noise ratio of $3 \sigma$, and a maximum radius of $10 \mathrm{arcsec}$. The 15 sources with a signal-to-noise ratio between 3 and 5 suggest that the HerBS sources might have two false detections. The overlay graph shows the position of the SCUBA-2 observation, where each point was centred on the SPIRE position.

cropped the observation to a $4 \times 4 \operatorname{arcmin}^{2}$ image, and measured the fluxes by measuring the highest flux density pixel in the central $50 \times 50 \operatorname{arcsec}^{2}$ region around the SPIRE-estimated position. We determine a SCUBA-2 detection by a combination of proximity to the Herschel-SPIRE $250 \mu \mathrm{m}$ position and the signal-to-noise ratio, as shown in Section 3.

\section{RESULTS}

We observed 203 of our pre-selected H-ATLAS sources with the SCUBA-2 instrument. In the following analysis, we find that 14 detected sources turn out to be blazars, which leaves our entire HerBS galaxy sample containing 209 sources. 152 of these sources are detected, 27 sources are not detected due to a signal-to-noise ratio cut and 10 sources do have a $3 \sigma$ detection, but not within the 10 arcsec circle around the SPIRE position. These results are summarized in Table 2.

Fig. 3 shows the distribution of the maximum signal-to-noise ratio in a $50 \times 50 \operatorname{arcsec}^{2}$ box centred on the SPIRE position, as a function of the position offset.

We decide to define a detected source by a signal-to-noise ratio greater than 3 and a positional offset smaller than 10 arcsec. Initially, we find 159 sources that satisfy this criterion, 27 sources that are not detected by the signal-to-noise ratio cut and 17 sources whose positional offset was too large.
Table 3. Re-examined SCUBA-2 observations of HerBS sources with $\theta>10 \operatorname{arcsec}$.

\begin{tabular}{lccc}
\hline HerBS & $\begin{array}{c}\theta \\
(\operatorname{arcsec})\end{array}$ & S/N & $\begin{array}{c}S_{850 \mu \mathrm{m}} \\
(\mathrm{mJy})\end{array}$ \\
\hline $\mathbf{6 3}$ & $\mathbf{9 . 4 5}$ & $\mathbf{3 . 1 9}$ & $\mathbf{3 3 . 8}$ \\
$\mathbf{7 5}$ & $\mathbf{7 . 5 9}$ & $\mathbf{4 . 2 4}$ & $\mathbf{4 4 . 9}$ \\
96 & 7.84 & 2.10 & 19.5 \\
97 & 6.57 & 2.49 & 28.1 \\
$\mathbf{1 0 1}$ & $\mathbf{1 . 9 3}$ & $\mathbf{3 . 4 2}$ & $\mathbf{3 2 . 5}$ \\
118 & 2.28 & 2.12 & 23.3 \\
122 & 6.97 & 2.43 & 21.9 \\
131 & 5.54 & 2.95 & 30.3 \\
$\mathbf{1 4 0}$ & $\mathbf{7 . 1 4}$ & $\mathbf{3 . 5 9}$ & $\mathbf{3 0 . 3}$ \\
$\mathbf{1 4 5}$ & $\mathbf{9 . 5 9}$ & $\mathbf{3 . 1 7}$ & $\mathbf{3 3 . 0}$ \\
146 & 7.85 & 2.92 & 32.1 \\
$\mathbf{1 4 8}$ & $\mathbf{5 . 4 0}$ & $\mathbf{3 . 0 2}$ & $\mathbf{2 9 . 0}$ \\
151 & 6.33 & 2.34 & 23.9 \\
163 & 6.66 & 1.85 & 19.1 \\
172 & 5.92 & 1.40 & 13.7 \\
$\mathbf{1 8 1}$ & $\mathbf{4 . 0 6}$ & $\mathbf{3 . 8 1}$ & $\mathbf{3 2 . 9}$ \\
195 & 3.94 & 2.61 & 29.5 \\
\hline
\end{tabular}

For each of the 17 sources that do not have their maximum flux within the 10 arcsec circle around the SPIRE position, which do have a signal-to-noise ratio greater than 3 , we decreased the size of the searching box to find the peak in flux. Of these 17 sources, seven sources have fluxes within 10 arcsec from the SPIRE position with a signal-to-noise ratio greater than 3 , as shown in boldface in Table 3. These seven sources are added to the detected sources.

Of the sources with signal-to-noise ratios between 3 and 5, 15 are originally situated outside of the 10 arcsec circle. These sources are distributed over 89 per cent of the map (the area outside the 10 arcsec circle). An even distribution of such false detections would result in two $(\sim 1.7)$ false detections inside the HerBS catalogue. The overlay graph inside Fig. 3 shows a strong correlation for most points around the centre, however, all other non-detections appear uniformly scattered, making an even distribution likely.

We know from Negrello et al. (2007) that there is a risk that several of these sources are blazar contaminations. In order to find these contaminants, we plot their flux ratios in Fig. 4.

The top panel shows the flux ratios based on just Herschel fluxes. We plot $S_{500 \mu \mathrm{m}} / S_{250 \mu \mathrm{m}}$ versus $S_{350 \mu \mathrm{m}} / S_{250 \mu \mathrm{m}}$. The sources that lie very close to a known blazar (within $10 \operatorname{arcsec}$ ) in the NASA Extragalactic Database (NED) (black circles) lie in the same region as the high-redshift HerBS sources (grey triangles, blue squares and red circles). We also plot the track for the template we derive in Section 4 through the diagram as the redshift changes (black line and circles). Similarly, we show the expected blazar track (assuming synchrotron radiation) for various possible $\alpha$-values (black dash-dot line and triangles). Note that both these tracks do not differ significantly from each other. The bottom panel shows the flux ratios of the 203 sources with SCUBA-2 observations. We plot $S_{850 \mu \mathrm{m}} / S_{250 \mu \mathrm{m}}$ against $S_{350 \mu \mathrm{m}} / S_{250 \mu \mathrm{m}}$. Most of the galaxies close to a known blazar occupy a different region of the graph, and can be easily identified and removed from the sample.

One of the sources, HerBS-16, does not have the typical flux ratios of a blazar, and has therefore not been removed. The spectrum also looks dust-like, and has consistent photometric redshift estimates, as can be seen in Fig. 5. The source, in this case, could be close to the blazar by accident. Only one source close to a known blazar 


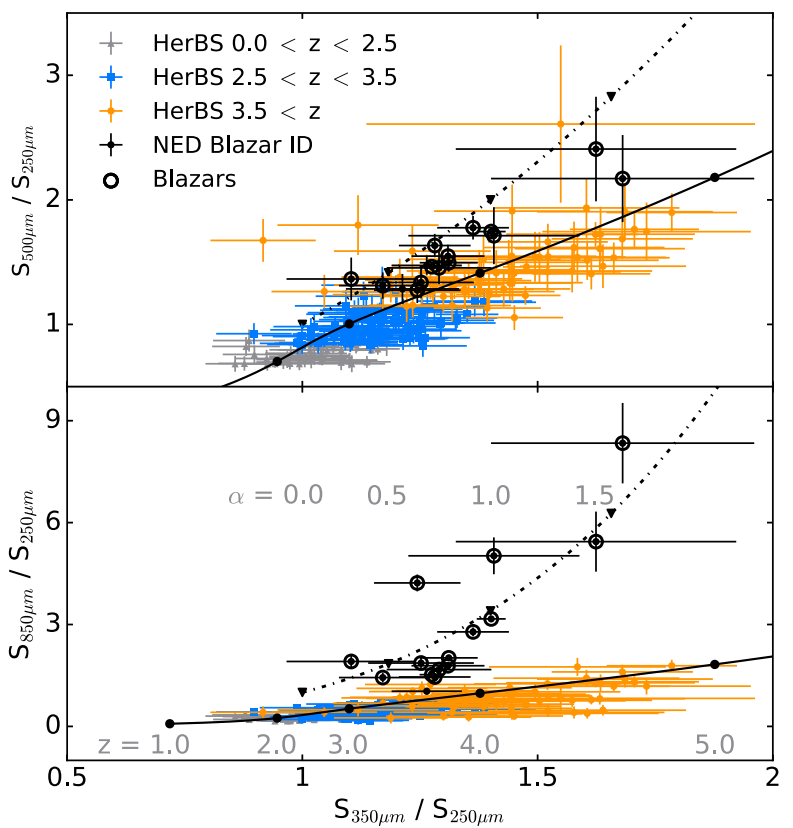

Figure 4. The top panel shows the flux ratios based on just Herschel fluxes. We plot $S_{500 \mu \mathrm{m}} / S_{250 \mu \mathrm{m}}$ versus $S_{350 \mu \mathrm{m}} / S_{250 \mu \mathrm{m}}$. Sources close to a known blazar in NED (black circles) lie in the same region as the high-redshift HerBS sources (grey triangles, blue squares and red circles). The bottom panel shows the flux ratios when we include the SCUBA-2 observations. We plot $S_{850 \mu \mathrm{m}} / S_{250 \mu \mathrm{m}}$ against $S_{350 \mu \mathrm{m}} / S_{250 \mu \mathrm{m}}$. Most sources close to a known blazar occupy a different region of the graph, and can be easily identified and removed (black circles). The difference between the graphs indicates the necessity of the $850 \mu \mathrm{m}$ observations for removing blazar contaminants from the sample. We also plot the track for the template we derive in Section 4 through the diagram as the redshift changes (black line and circles). Similarly, we show the expected blazar track for $\alpha$-values ranging from 0 to 1.5 (black dash-dot line and triangles).

has not been observed, and we have therefore kept it in our HerBS sample (HerBS-112).

The difference between the graphs indicates the need for multiwavelength observations, in order to reliably remove blazar contaminants from the sample. We list the Herschel SPIRE and SCUBA-2 positions and fluxes of the removed blazars in Table A2.

After removing 14 blazars from our sample, we are left with 189 HerBS galaxies with SCUBA-2 observations. While some sources close to NED blazars did not have irregular flux ratios, all of the sources with irregular flux ratios are close to known blazars. This suggests our method for finding contaminants in our sample is robust, and thus that the 19 unobserved sources that do not lie close to a NED blazar are not likely to have emission dominated by synchrotron radiation.

For completeness, we plot the blazar spectrum, assuming solely synchrotron radiation, in Fig. 4, following equation:

$S_{v}=A v^{-\alpha}$.

Here $S_{v}$ is the flux density at a specific frequency ( $\left.v\right), A$ is a constant factor and $\alpha$ determines the steepness of the slope in the far-infrared wavelength regime. Most of the blazars lie close to this line. We also calculate the value for $\alpha$ for each galaxy, by minimizing $\chi^{2}$ :

$\chi^{2}=\sum^{i>j}\left[\frac{\left(S_{i} / S_{j}\right)_{\text {model }}-\left(S_{i} / S_{j}\right)_{\text {meas }}}{\sigma_{i, j \text {, meas }}}\right]^{2}$.

The index $i$ and $j$ iterate over all four wavelengths (250, 350, 500 and $850 \mu \mathrm{m}$ ), where $i$ 's wavelength is always larger than $j . \sigma_{i, j \text {, meas }}$ is the combined error of $\left(S_{i} / S_{j}\right)_{\text {meas }} . \alpha$-values range from 0.24 to 1.66. The individual values can be found in Table A2, and agree well with the positions of the blazar sources in Fig. 4.

We provide postage stamp cutouts of the observations with SPIRE, SCUBA-2 and fits of our templates (Section 4.1) to the 250, 350, 500 and $850 \mu \mathrm{m}$ flux densities of each source in Appendix B. Typical cutouts of a source detected by SCUBA-2, a source undetected by SCUBA-2 and a blazar are shown in Fig. 5. The bottom row of cutouts shows HerBS-16, which is close to a NED blazar, but has a spectral energy distribution (SED) typical of a submm galaxy.

\section{GALAXY TEMPLATES}

We derived a galaxy template for our total sample by using the subset of HerBS sources that have spectroscopic redshifts. We fitted a two-temperature, modified blackbody SED to the Herschel and the SCUBA-2 flux densities of each source. We list the sources with spectroscopic redshifts in Table 4. These spectroscopic redshifts were found by observing submm spectral lines, in order to ensure we are looking at the same source.

This template is necessary to estimate photometric redshifts and luminosities for our entire sample. Similar to the analysis of Pearson et al. (2013), we fitted the template to the SPIRE (250, 350 and $500 \mu \mathrm{m}$ ) fluxes, and included our JCMT/SCUBA-2 $850 \mu \mathrm{m}$ flux densities. We choose to exclude the PACS photometry of our sources in our analysis, as even the brightest sources are poorly detected, due to the high-redshift limit of our sample. Our spectroscopic sample includes eight sources used in Pearson's analysis, and 16 new sources, all of which are at high redshifts $\left(z_{\text {spec }}>1.5\right)$. We only used HerBS sources for our template to ensure there is $850 \mu \mathrm{m}$ photometry of our sources, and only used the galaxies with spectroscopic redshifts estimated from more than one line.

\subsection{Template fitting}

We fitted the template to the sources' flux densities and rest wavelengths, calculated from their spectroscopic redshifts. We assumed a two-temperature modified blackbody template for the SED,

$S_{v}=A_{\text {off }}\left[B_{v}\left(T_{\mathrm{h}}\right) v^{\beta}+\alpha B_{v}\left(T_{\mathrm{c}}\right) v^{\beta}\right]$,

where $S_{v}$ is the flux at the rest-frame frequency $v, A_{\text {off }}$ is the normalization factor, $B_{v}$ is the Planck blackbody function, $\beta$ is the dust emissivity index, $T_{\mathrm{h}}$ and $T_{\mathrm{c}}$ are the temperatures of the hot and cold dust components and $\alpha$ is the ratio of the mass of the cold to hot dust.

We aimed to minimize the following $\chi^{2}$ for the fluxes that were detected:

$\chi^{2}=\sum_{i=1}^{n} \chi_{i}^{2}=\sum_{i=1}^{n} \sum^{\lambda}\left[\frac{A_{i} S_{\text {model }, i}-S_{\text {meas }, i}}{\sigma_{\text {meas }, i}}\right]^{2}$,

where $S_{\text {model }, i}$ is the predicted flux of the $i$ th source (out of $n$ ) according to equation (3), with the amplitude $A_{\text {off }}$ set to $1 . S_{\text {meas }, i}$ and $\sigma_{\text {meas }, i}$ are the measured signal and noise values. In the case of all fluxes of the source were detected, we fitted the amplitude of our template, $A_{i}$, to the rest-wavelength data points analytically in order to decrease computation time,

$A_{i}=\left(\sum^{\lambda} \frac{S_{\text {model }, j} S_{\text {meas }, j}}{\sigma_{\text {meas }, j}^{2}}\right) /\left(\sum^{\lambda} \frac{S_{\text {model }, j}^{2}}{\sigma_{\text {meas }, j}^{2}}\right)$.

Equation (5) is derived by solving $\mathrm{d} \chi_{i}^{2} / \mathrm{d} A_{i}=0$. We left the one source with a spectroscopic redshift did not have a detected 


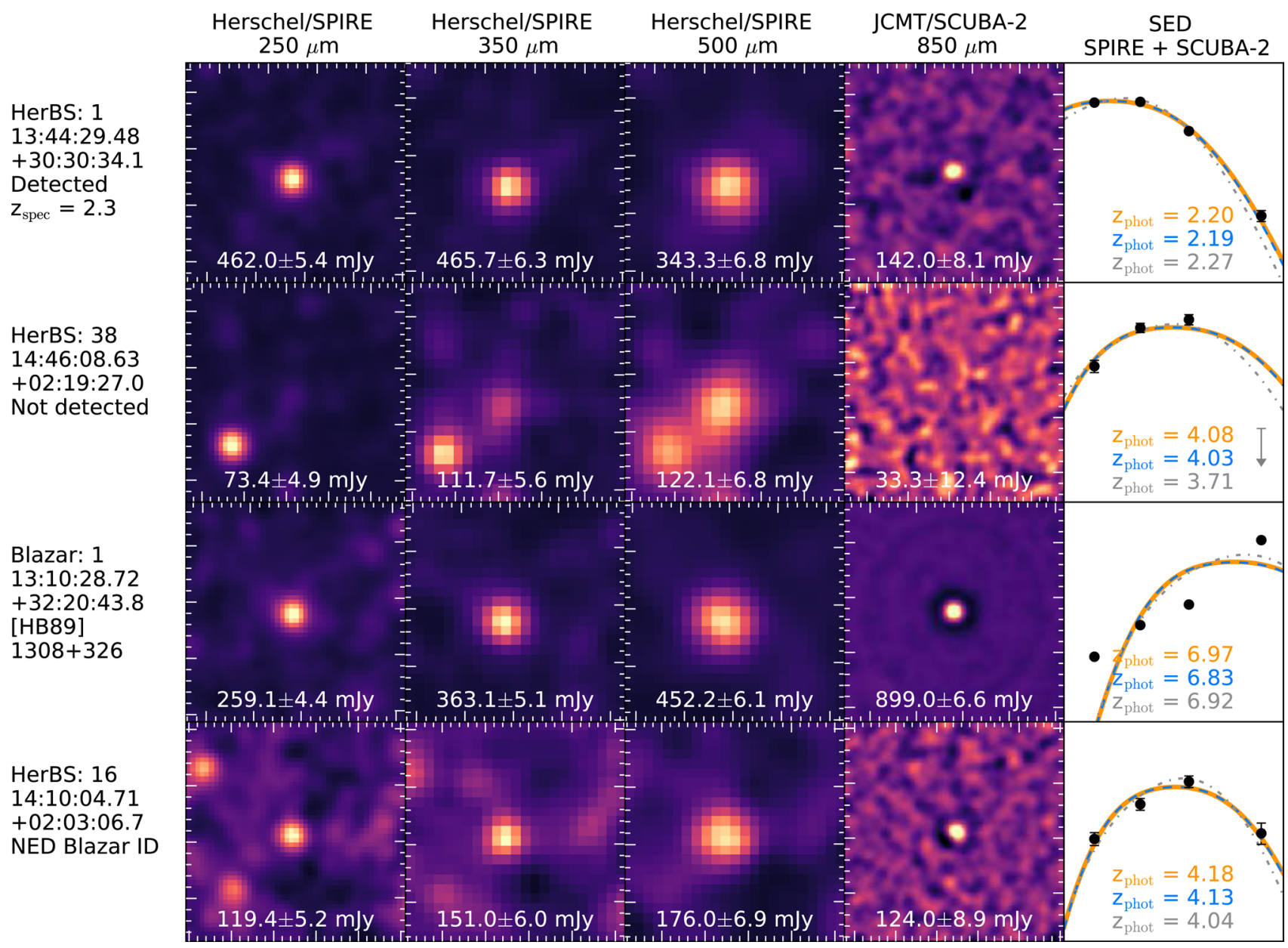

Figure 5. The four different types of sources we found in the SCUBA-2 $850 \mu \mathrm{m}$ observations of our sample: a galaxy detected with SCUBA-2, a galaxy undetected with SCUBA-2, a blazar and HerBS-16, which is close to a known blazar, but has an SED typical of thermal emission from dust. The first three columns of cutouts of each source are the Herschel observations shown in $4 \times 4 \operatorname{arcmin}^{2}$ postage stamps. The fourth column shows the $850 \mu \mathrm{m}$ SCUBA-2 observation in a $4 \times 4 \mathrm{arcmin}^{2}$ postage stamp. All postage stamps are centred at the $250 \mu \mathrm{m}$ extraction position of the Herschel catalogue. The final frame is a fitted SED, with the best-fitting template in orange, fixed $\beta$ template in blue and Pearson's template in grey (Pearson et al. 2013). Similar figures for the entire HerBS sample can be found in Appendix B.

SCUBA-2 flux, HerBS-71. In this upper limit case, we calculated the $\chi^{2}$ contribution using the method detailed in Sawicki (2012) and Thomson et al. (2017):

$\chi^{2}=-2 \sum_{j} \ln \int_{-\infty}^{3 \sigma} \exp \left[-\frac{1}{2}\left(\frac{f-A_{j} S_{\text {model }, j}}{\sigma_{\text {meas }, j}}\right)^{2}\right] \mathrm{d} f$,

where we sum over all non-detections $j$, which in our case is only the SCUBA-2 flux of HerBS-71, and integrate the Gaussian distribution up to the detection criterion of three times the measured noise $(3 \sigma)$. The modified $\chi^{2}$ statistic quantifies the probability of an event where the noise affected the signal to drop below the detection criterion. In the case of the model predicts a flux under the detection limit, there is no discrepancy with the model, and we set the $\chi^{2}$-value to zero.

We did this template fitting for two templates: best fit, where we varied all the parameters $\left(T_{\mathrm{c}}, T_{\mathrm{h}}, \alpha\right.$ and $\left.\beta\right)$; and fixed $\beta$, where we varied all parameters except $\beta$, which we fixed to 2 . We also tried keeping $T_{\mathrm{c}}, T_{\mathrm{h}}, \alpha$ and $\beta$ fixed to the values found by Pearson et al. (2013). In this case we found the set of $A_{i}$ that gave the minimum $\chi^{2}$ fit. The point of this was to determine whether our new templates gave any improvement in the quality of fit over that found by Pearson et al. (2013). We estimated the uncertainty on each parameter by incrementally changing this parameter until the minimized $\chi^{2}$ changes by of one (one interesting parameter; Avni 1976). The $\chi^{2}$ was minimized by allowing the other (two or three) parameters to vary. The best-fitting templates are given in Table 4.

\subsection{Template results}

We find a cold- and hot-dust temperature of $21.29_{-1.66}^{+1.35}$ and $45.80_{-3.48}^{+2.88} \mathrm{~K}$, a cold-to-hot dust mass ratio of $26.62_{-6.74}^{+5.61}$ and a $\beta$ of $1.83_{-0.28}^{+0.14}$ for the best-fitting template. The results for the other templates, including the fitting of the templates to redshift and luminosity subsets, can be found in Table 5 .

We investigated the usefulness of each template for estimating photometric redshifts, by using each template to estimate the photometric redshift of each source, and then calculating $\left(z_{\text {spec }}-z_{\text {phot }}\right) /\left(1+z_{\text {spec }}\right)$ for each source. The root mean squared value of $\left(z_{\text {spec }}-z_{\text {phot }}\right) /\left(1+z_{\text {spec }}\right)$ for the best-fitting template is 13 per cent, which is similar to the fixed $\beta$ and Pearson templates. The value of the relative error derived from the best-fitting template 
Table 4. The sources from the HerBS sample with measured spectroscopic redshifts.

\begin{tabular}{lccccc}
\hline H-ATLAS name & HerBS & $z_{\text {spec }}$ & $z_{\text {phot }}$ & $\Delta z /(1+z)$ & Ref. \\
\hline \multicolumn{5}{c}{ Robust, multi-line detections } \\
J083518.4+303034 & 1 & 2.30 & 2.20 & 0.03 & H12 \\
J114637.9-001132 & 2 & 3.26 & 2.80 & 0.11 & H12 \\
J082403.8+334407 & 3 & 2.95 & 3.75 & -0.20 & H-p \\
J083051.0+013225 & 4 & 3.63 & 3.09 & 0.12 & R-p \\
J080520.2+233627 & 5 & 3.57 & 3.72 & -0.03 & R-p \\
J082246.8+284449 & 6 & 1.68 & 2.11 & -0.16 & G13 \\
J082537.0+292326 & 7 & 2.78 & 2.89 & -0.03 & K-p \\
J084933.4+021442 & 8 & 2.41 & 2.64 & -0.07 & L-p \\
J080214.5+261457 & 9 & 3.68 & 3.87 & -0.04 & K-p \\
J113526.2-014606 & 10 & 3.13 & 2.32 & 0.20 & H12 \\
J082620.3+245900 & 12 & 3.11 & 2.29 & 0.20 & R-p \\
J142413.9+022303 & 13 & 4.28 & 4.53 & -0.05 & C11 \\
J141351.9-000026 & 15 & 2.48 & 2.62 & -0.04 & H12 \\
J090311.6+003907 & 19 & 3.04 & 3.76 & -0.18 & F11 \\
J082310.2+311534 & 20 & 1.84 & 1.88 & -0.02 & R-p \\
J083144.0+255054 & 29 & 2.34 & 2.69 & -0.11 & R-p \\
J082153.5+341649 & 30 & 2.19 & 3.28 & -0.34 & R-p \\
J091840.8+023048 & 32 & 2.58 & 3.03 & -0.13 & H12 \\
J082949.3+300401 & 35 & 2.68 & 2.73 & -0.01 & H-p \\
J091304.9-005344 & 59 & 2.63 & 2.87 & -0.07 & N10 \\
J115820.1-013752 & 66 & 2.19 & 2.49 & -0.09 & H-p \\
J113243.0-005108 & 71 & 2.58 & 3.73 & -0.32 & R-p \\
& & & & &
\end{tabular}

Tentative, single-line detections (not used)

$\begin{array}{lccccc}\text { J080532.7+275900 } & 31 & 2.79 & 3.25 & -0.12 & - \\ \text { J083344.9+000109 } & 88 & 3.10 & 3.25 & -0.04 & - \\ \text { J113803.6-011737 } & 96 & 3.15 & 2.88 & -0.07 & \text { H12 } \\ \text { J113833.3+004909 } & 100 & 2.22 & 2.66 & -0.14 & -\end{array}$

Note. Reading from the left, the columns are: column 1 - the official $\mathrm{H}-$ ATLAS name; column 2 - HerBS number; column 3 - spectroscopic redshift; column 4 - photometric redshift using the best-fitting model; column $5-\left(z_{\text {spec }}-z_{\text {phot }}\right) /\left(1+z_{\text {spec }}\right)$; column 6 - reference for the spectroscopic redshift: N10 - Negrello et al. (2010); F11 - Frayer et al. (2011); H12 Harris et al. (2012); G13 - George et al. (2013); L13 - Lupu et al. (2012); B13 - Bussmann et al. (2013); H-p - Harris et al. (in preparation); R-p Riechers et al. (in preparation); K-p - Krips et al. (in preparation); L-p Lupu et al. (in preparation).

Table 5. The results of the fitting of the total sample, with a variable and fixed beta, and applying the template from Pearson et al. (2013) to our sources.

\begin{tabular}{lccc}
\hline & Total & Fixed $\beta$ & Pearson \\
\hline$T_{\mathrm{c}}(\mathrm{K})$ & $21.29_{-1.66}^{+1.35}$ & $20.47_{-0.26}^{+0.26}$ & 23.9 \\
$T_{\mathrm{h}}(\mathrm{K})$ & $45.80_{-3.48}^{+2.88}$ & $44.05_{-0.55}^{+0.52}$ & 46.9 \\
$\alpha$ & $26.69_{-6.74}^{+5.61}$ & $30.46_{-1.42}^{+1.32}$ & 30.1 \\
$\beta$ & $1.83_{-0.28}^{+0.14}$ & 2 (fixed) & 2 (fixed) \\
$\chi^{2}$ & 812.58 & 812.96 & 1101.03 \\
$\Delta z /\left(z_{\text {spec }}+1\right)$ & $-0.03 \pm 0.14$ & $-0.03 \pm 0.14$ & $-0.01 \pm 0.12$ \\
\hline
\end{tabular}

for each source is given in Table 4, and the mean and standard deviations of this quantity for each template are given in Table 5.

Fig. 6 shows $\left(z_{\text {spec }}-z_{\text {phot }}\right) /\left(1+z_{\text {spec }}\right)$ plotted against spectroscopic redshift for the three templates. The three distributions are very similar. We compare the redshift estimates against the method used in Ivison et al. (2016). They fit three different tem-

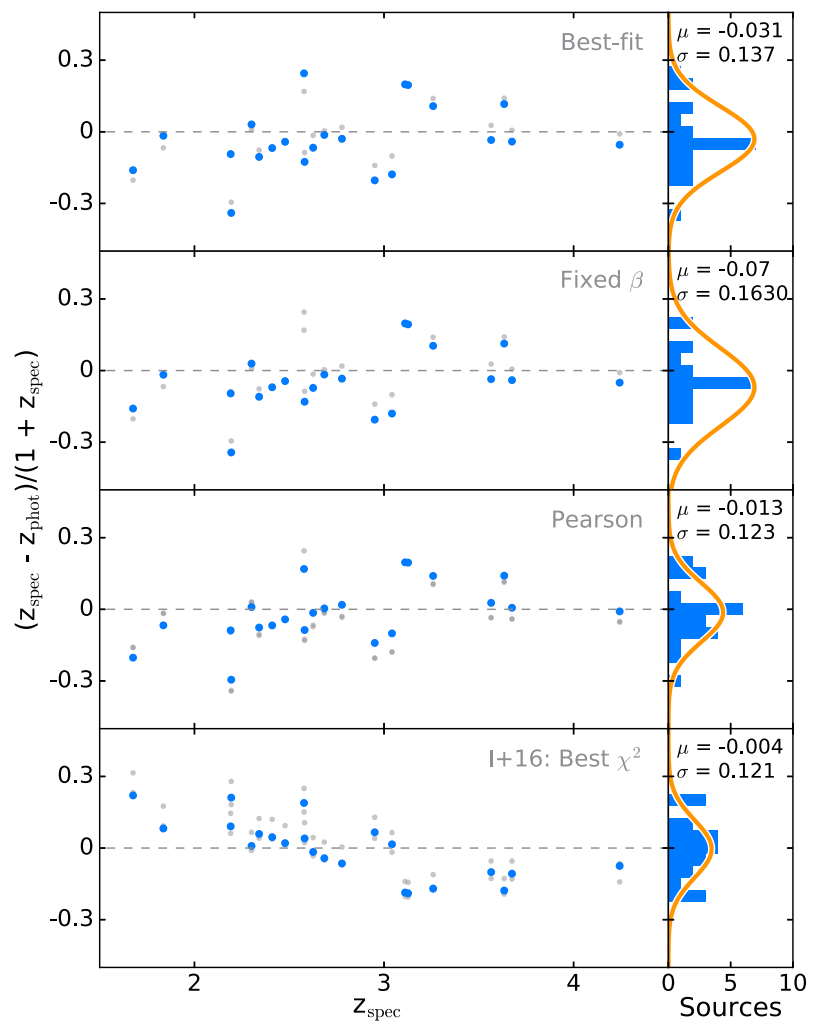

Figure 6. The top three panels show $\left(z_{\text {spec }}-z_{\text {phot }}\right) /\left(1+z_{\text {spec }}\right)$ plotted against the spectroscopic redshift for the three templates. The blue dots in each panel show the points for the specified template, while the smaller grey dots show the points for the other two templates. The bottom panel shows $\left(z_{\text {spec }}-z_{\text {phot }}\right) /\left(1+z_{\text {spec }}\right)$ for the three templates used for the redshift estimation in Ivison et al. (2016), where the blue dots correspond to the template fit with the lowest $\chi^{2}$ for each source individually, and the smaller grey dots are the values of the two remaining templates.

plates [ALESS (Swinbank et al. 2014), Cosmic Eyelash (Ivison et al. 2010; Swinbank et al. 2010) and the template from Pope et al. (2008)] to the flux measurements, and use the redshift estimate from the spectrum with lowest $\chi^{2}$-value. When we apply this method to our sample of sources with spectroscopic redshifts, we achieve a slightly better redshift accuracy of $\sim 12$ per cent.

We note that the uncertainty in photometric redshift estimation using our new template, obtained from SCUBA-2 and Herschel measurements, is not actually any smaller than that using the template that Pearson et al. (2013) obtained from Herschel measurements alone. We discuss the significance of this in the Section 5.

Fig. 7 shows the normalized flux densities of the spectroscopic sources against their rest-frame wavelength, with the three templates overlaid. The flux densities are normalized to give each galaxy the same bolometric luminosity as HerBS-1.

We used the photometric redshifts estimates of our best-fitting template to derive observed bolometric luminosities of the HerBS sources. As the redshift estimates are determined from a different spectrum, some of the photometric redshift estimates, $z_{\text {phot }}$, fall below 2. They are, however, kept in the HerBS sample, as not to increase the complexity of the selection functions.

We calculate the observed bolometric luminosities by deriving the photometric redshift from our best-fitting template, and integrating the template from $\lambda_{\text {rest }}=8$ to $1000 \mu \mathrm{m}$. The estimated redshifts and bolometric luminosities are listed in Table A1, as well as the 


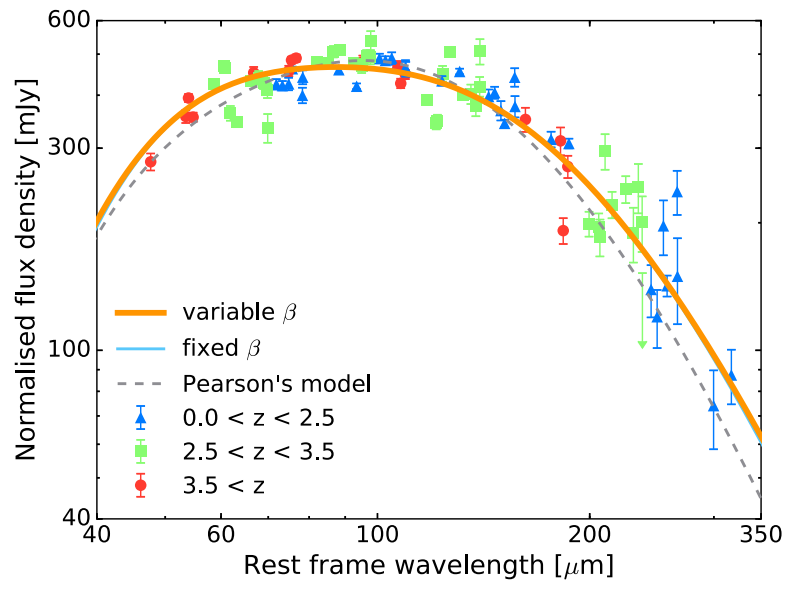

Figure 7. The flux densities of the spectroscopic sources plotted against rest-frame wavelength. The curves show the three templates (best fit is the thick orange line, fixed $\beta$ is the thin blue line and Pearson's model is the dashed grey line), and all the flux densities of each source are scaled to produce the same bolometric luminosity as the brightest source (HerBS: 1). The sample is split up in three redshift intervals, to associate each galaxy's four data points more easily.

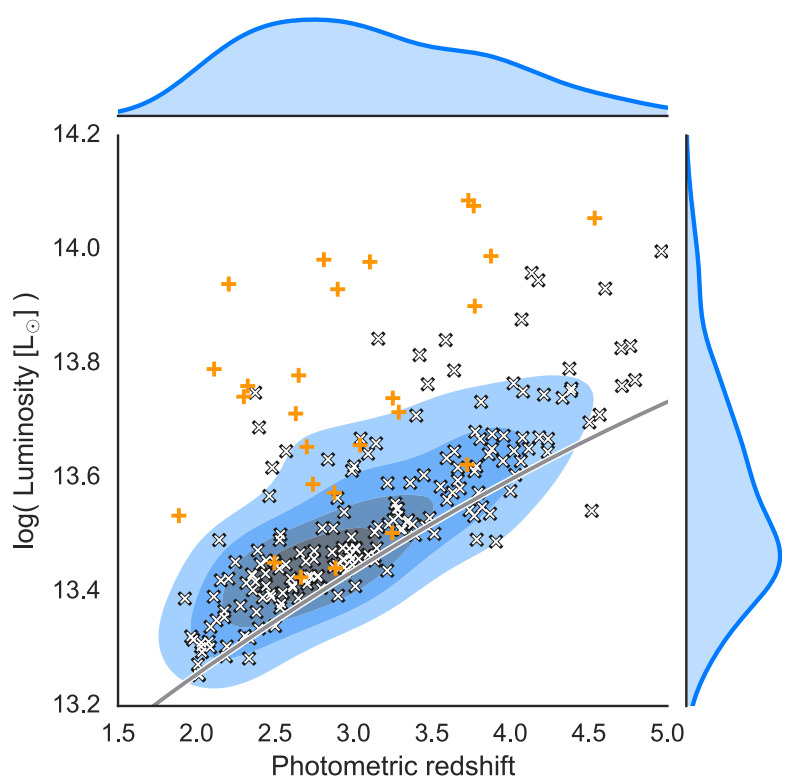

Figure 8. Observed bolometric far-infrared luminosity $\left(\lambda_{\text {rest }}=8-1000 \mu \mathrm{m}\right)$ plotted against photometric redshift, calculated with the best-fitting template. Sources with spectroscopic redshifts are plotted in orange plusses, although the redshifts used in the diagram are their photometric redshifts. The smoothed distributions of redshift and luminosity are shown on the sides of the scatter plots. The grey line shows bolometric luminosity for the best-fitting template, assuming $S_{500 \mu \mathrm{m}}=80 \mathrm{mJy}$, as a function of redshift.

photometric redshift estimates using the method from Ivison et al. (2016). Fig. 8 shows the distribution of sources as a function of redshift and luminosity. This figure shows that the majority of our sources with a spectroscopic redshift are in the higher luminosity range, as typically spectroscopic campaigns aim for the brightest sources first.

\section{DISCUSSION}

\subsection{Source confusion}

We have selected our HerBS sample using a $500 \mu \mathrm{m}$ flux limit. The large beam width at this wavelength could cause us to confuse multiple line-of-sight sources into a single observed source, and hence yield a $500 \mu \mathrm{m}$ flux density that is too large.

Observationally, high-resolution studies of sub-millimetre galaxies show this to be the case, although the severity of this effect varies from study to study (Hodge et al. 2013; Koprowski et al. 2014). An SMA study by Chen et al. (2013) of sources selected at $450 \mu \mathrm{m}$ only found 10 per cent of the sources to be significantly amplified by line-of-sight sources. An ALMA survey of $870 \mu \mathrm{m}$ selected ALESS sources finds that up to 50 percent of the sources are significantly affected (Hodge et al. 2013; Karim et al. 2013). Longer wavelengths and higher selection flux densities correlate with more source confusion, although all observational multiplicity studies so far focus on SMGs with a low probability of lensing.

A recent study by Scudder et al. (2016) used Bayesian inference methods to estimate the effects of source confusion in Herschel observations at $250 \mu \mathrm{m}$. They concluded that individual $250 \mu \mathrm{m}$ sources are often the combination of emission from more than one galaxy.

The solid angle of the beam of the JCMT at $850 \mu \mathrm{m}$ is six times smaller than the beam of the $500 \mu \mathrm{m}$ SPIRE observations. We do not see any of our HerBS sources resolve into multiple $>3 \sigma$-detected components. This suggests that our long-wavelength observations are not confused, unless the sources are clustered on a scale smaller than the JCMT's beam size. The small clustering size could be the case, as Karim et al. (2013) find the multiple emissions are separated less than 6 arcsec in the majority of cases of source confusion. Similarly, Chen et al. (2016) measured the clustering of SMGs on scales down to 1.5 arcsec using SCUBA-2 combined with deep nearinfrared and optical data, and they also report a steep increase in angular correlation below 6 arcsec. However, Hayward et al. (2013) simulated light cones to estimate the blending ratio of associated and unassociated SMGs for a 15 arcsec beam, and found that at least 50 per cent of all blended SMGs show an unassociated SMG. The HerBS sources are selected by their $500 \mu \mathrm{m}$ flux, which has a 36 arcsec beam, and should therefore be more influenced by unassociated SMGs. As these unassociated SMGs are spatially unrelated to the source, they should have shown up in our JCMT analysis. A reason for the lack of source confusion could be due to our selection of lensed sources, as the probability for gravitational lensing is small, and two unrelated sources in the same Herschel beam are unlikely to be both lensed by the same galaxy.

Strong gravitational lensing could also be caused by a cluster of galaxies, which acts on a longer angular scale. These events are less common (Negrello et al. 2017), however Zavala et al. (2015) did report on the redshifts of cluster-lensed sources, one of which turned out to be three sources that was blended and lensed. We did not exclude these possibilities, however considering their infrequency, we can state that this lensing type would not influence the entire sample.

\subsection{The diversity of galaxies}

In Section 4, we fitted a two-temperature modified blackbody template to 22 HerBS sources with spectroscopic redshifts, the results of which can be seen in Table 5 . 
Both the fixed- $\beta$ and best-fitting templates result in similar templates, as the $\beta$-value of the best-fitting template is similar within the error bars. The errors on the best-fitting template are slightly larger, as more parameters are being fitted. The temperatures on both fitted templates are slightly cooler than the template from Pearson et al. (2013); however, we do not find an indication of a cool gas component with a temperature $T<20 \mathrm{~K}$, as found in Planck Collaboration XVI (2011) and Clements, Dunne \& Eales (2010). The values we find for the temperatures agree broadly with the initial fitting attempts by Dunne \& Eales (2001), and the overall findings of Clements et al. (2010).

The large $\chi^{2}$ values in Table 5 imply that a single template is not actually a good representation of the data. We fit our template to 22 galaxies, each with four data points, except one source where we only fitted the three SPIRE fluxes, as its SCUBA-2 flux remained undetected. The free parameters in our model are the template parameters (three or four) and the amplitudes for each galaxy (22, equation 5). The expected $\chi^{2}$ values for the two models, on the assumption that they are a good representation of the data, are therefore

$$
\begin{aligned}
\chi_{\text {best-fit }}^{2} & \approx N_{\text {data }}-N_{\text {param }}-1 \\
& \approx 4 \times 22-22-4-1 \\
& \approx 61, \\
\chi_{\text {fixed } \beta}^{2} & \approx N_{\text {data }}-N_{\text {param }}-1 \\
& \approx 4 \times 22-22-3-1 \\
& \approx 62 .
\end{aligned}
$$

However, we observe $\chi^{2}$-values of $\sim 812$, indicating that our sources are poorly modelled by a single galaxy template.

We tested the photometric redshift estimates of the templates using the same sources we used to derive the best-fitting template. However, we found no improvement in accuracy (Table 5) compared to the older template of Pearson et al. (2013). Similarly, Fig. 6 shows a similar pattern of redshift errors for all three templates. The redshift estimation by Ivison et al. (2016) might provide a slightly better estimation of the redshift, which are therefore added to the catalogue (Table A1). The explanation for this lack of improvement is almost certainly the diversity of the population; the limit on the accuracy of photometric redshift estimates is not set by the accuracy of the average template but by the fact that galaxies have different SEDs.

\subsection{Redshift distribution of the HerBS sample}

Fig. 9 shows the redshift distribution of the HerBS sample, compared against various other galaxy samples that are summarized in Table 6. The top panel compares the distribution to samples selected with a simple flux cut-off at $500 \mu \mathrm{m}$. The sample from $\mathrm{Ne}-$ grello et al. (2017) used a $S_{500 \mu \mathrm{m}}>100 \mathrm{mJy}$ flux cut on $600 \mathrm{deg}^{2}$ of the H-ATLAS field (they used a conservative mask on the SGP field). The sample from Nayyeri et al. (2016) used the same flux cut on the $372 \mathrm{deg}^{2} \mathrm{HeLMS}$ and HeRS fields. We plot the total sample from Wardlow et al. (2013). They used the $95 \mathrm{deg}^{2}$ HerMES survey, and their $500 \mu \mathrm{m}$ flux cut-off went down to $80 \mathrm{mJy}$.

The bottom panel compares the HerBS redshift distribution against samples selected at various wavelengths. The sample from Ivison et al. (2016) is also from the H-ATLAS fields, and contains sources with a colour cut at $S_{500 \mu \mathrm{m}} / S_{250 \mu \mathrm{m}}>1.5$ and $S_{500 \mu \mathrm{m}} / S_{350 \mu \mathrm{m}}>0.85$, in order to select sources at high redshift. The sources were also selected to have relatively low $500 \mu \mathrm{m}$ flux

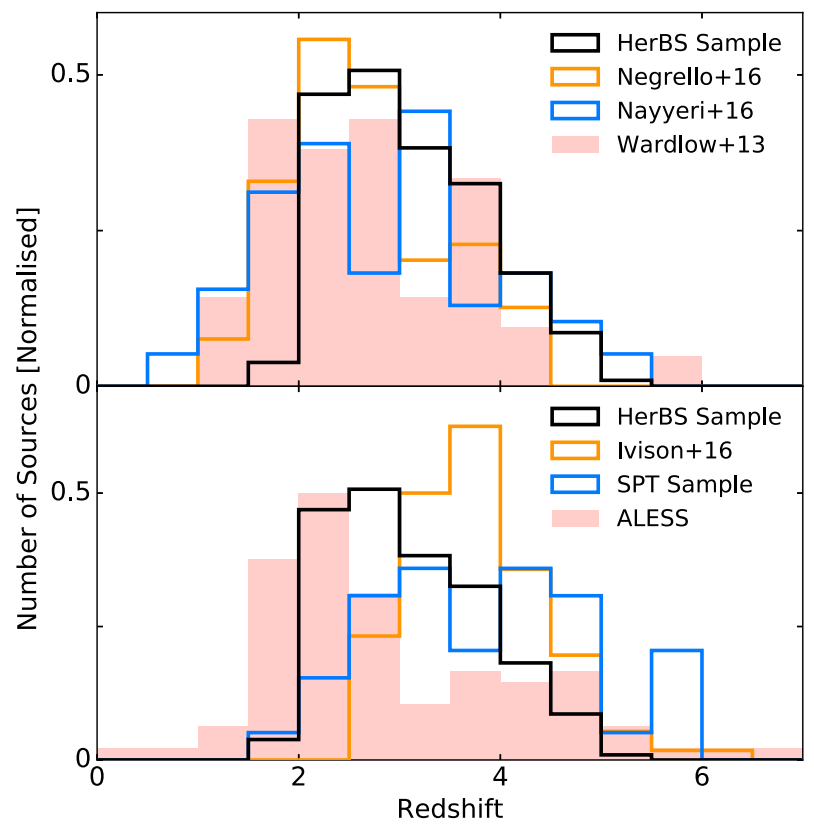

Figure 9. The top panel compares the redshift distribution of the HerBS sample (black) to that of three samples selected with Herschel/SPIRE at $500 \mu \mathrm{m}$. The bottom panel compares the redshift distribution of the HerBS sample (black) to that of three samples with different selection wavelengths and colour cuts.

density of around $50 \mathrm{mJy}$, in order to select unlensed sources. Their unlensed nature reduces the uncertainty in the intrinsic luminosity of the source. The South Pole Telescope (SPT) lensed sample was selected from $2500 \mathrm{deg}^{2}$ SPT survey by a flux cut at $\mathrm{S}_{1.4 \mathrm{~mm}}>20 \mathrm{mJy}$, and demanding the source has a dust-like spectrum. Low-redshift sources were removed with radio and far-infrared flux limits (Weiß et al. 2013; Strandet et al. 2016). The ALESS sample is initially selected from the LESS sample at $S_{870 \mu \mathrm{m}}>4.4 \mathrm{mJy}$ from the $0.25 \mathrm{deg}^{2}$ Extended Chandra Deep Field-South (ECDFS) field (Weiß et al. 2009). ALMA observations of the LESS sample removed all contaminants, resulting in a final ALMA-LESS (ALESS) sample of 96 SMGs (Simpson et al. 2014).

All samples selected at $500 \mu \mathrm{m}$ with a simple flux cut have a similar redshift profile, and do not differ significantly from the HerBS sample when we take the photometric redshift cut-off into account. Also, without the photometric redshift cut-off, the standard deviation of the HerBS sample would have been larger.

Typically, higher average redshifts are expected for longer selection wavelengths (Bethermin et al. 2015). We see this for the SPT sample, which has higher average redshifts. The ALESS sample, selected at $870 \mu \mathrm{m}$, has a higher average redshift than the $500 \mu \mathrm{m}$ without redshift constraints, but a lower average redshift than the HerBS sample due to HerBS photometric redshift constraint. The SPT and ALESS samples have a larger standard deviation in their redshifts, because the $K$-correction is negative for wavelengths between $850 \mu \mathrm{m}$ and $\sim 3 \mathrm{~mm}$. Comparison with the Ivison's sample is difficult because of the more complicated selection criteria they employ.

A way of quantifying the similarity between the samples is using the Kolmogorov-Smirnov (KS) test. We compare each sample's sources with a redshift (spectroscopically or photometrically determined) greater than 2 to the photometric redshifts of the HerBS sources with $z_{\text {phot }}>2$. For each sample, we run this method 100000 times while randomly varying the redshift of each source 
Table 6. Redshift distributions of several submm samples.

\begin{tabular}{lccccl}
\hline Sample & $\langle z\rangle \pm \sigma$ & Sources & Surface & KS $\sigma$-value & Selection criterion \\
\hline HerBS & $3.09 \pm 0.71$ & 209 & 616.4 & $1.27 \pm 0.45$ & $S_{500 \mu \mathrm{m}}>80 \mathrm{mJy} ; z_{\text {phot }}>2.0$ \\
HerBS with $z_{\text {spec }}$ & $3.07 \pm 0.72$ & 22 & 616.4 & $2.01 \pm 0.31$ & $S_{500 \mu \mathrm{m}}>80 \mathrm{mJy} ; z_{\text {phot }}>2.0$ \\
Negrello & $2.64 \pm 0.75$ & 80 & 616.4 & $1.82 \pm 0.77$ & $S_{500 \mu \mathrm{m}}>100 \mathrm{mJy}$ \\
Nayyeri & $2.77 \pm 1.02$ & 77 & 372 & $0.66 \pm 0.50$ & $S_{500 \mu \mathrm{m}}>100 \mathrm{mJy}$ \\
Wardlow & $2.65 \pm 0.90$ & 42 & 95 & $0.93 \pm 0.66$ & $S_{500 \mu \mathrm{m}}>80 \mathrm{mJy}$ \\
Ivison & $3.80 \pm 0.67$ & 112 & 616.4 & $2.31 \pm 0.84$ & $S_{500 \mu \mathrm{m}} \sim 50 \mathrm{mJy} ; S_{500 \mu \mathrm{m}} / S_{250 \mu \mathrm{m}}>1.5 ; S_{500 \mu \mathrm{m}} / S_{350 \mu \mathrm{m}}>0.85$ \\
SPT sample & $3.81 \pm 1.07$ & 39 & 2500 & $0.88 \pm 0.55$ & $S_{1.4 \mathrm{~mm}}>20 \mathrm{mJy}$ \\
ALESS & $2.90 \pm 1.22$ & 96 & 0.25 & $1.26 \pm 0.54$ & $S_{870 \mu \mathrm{m}}>4.4 \mathrm{mJy}$ \\
\hline
\end{tabular}

according to a Gaussian distribution with a width of $\Delta z=0.15(1$ $+z$ ). For the comparison to Ivison's sample, we only compare it to HerBS sources with a similar colour cut as they employed $\left(S_{500 \mu \mathrm{m}} / S_{250 \mu \mathrm{m}}>1.5\right.$ and $\left.S_{500 \mu \mathrm{m}} / S_{350 \mu \mathrm{m}}>0.85\right)$, which only 26 HerBS sources follow. For the SPT sample, we used our bestfitting template to estimate the flux at $1.4 \mathrm{~mm}$, and only compared the sources that follow the SPT flux cut $\left(S_{1.4 \mathrm{~mm}}>20 \mathrm{mJy}\right)$, a property only 60 HerBS sources have. The ALESS flux criterion $\left(S_{870 \mu \mathrm{m}}>4.4 \mathrm{mJy}\right)$ was also estimated using the best-fitting template, and was met by all our 209 sources.

We detail the KS probability values in terms of disagreement between two samples in standard deviations $(\sigma)$ in Table 6 . A comparison between the redistributed redshifts and the original, unvaried redshift estimates of the HerBS sources gives a $1.27 \pm 0.45$ times the standard deviation, which indicates we should expect rather large uncertainties in the probability measurements. The spectroscopic redshifts of the HerBS sources disagree with $2.01 \pm 0.31$ times the standard deviation with the redistributed redshifts. When we compare the photometric redshift estimates of these spectroscopic sources to the HerBS sample, this value drops to $0.79 \pm 0.56$. Our HerBS sample thus appears probed evenly by the current set of HerBS sources with spectroscopic redshifts.

The sample from Negrello features more galaxies at low selected redshifts $(2<z<3)$, causing the disagreement seen by the relatively high KS value. This is contrary to both Nayyeri and Wardlow's samples, who agree strongly with the HerBS sample, suggesting that these sources are drawn from the same population. Only one out of four sources with low $500 \mu \mathrm{m}$ flux densities ( $~ 80 \mathrm{mJy})$ in Wardlow's sample was found to be lensed. This seems contradictory to the high likeness with the HerBS sample, which has a high lensing fraction of 76 per cent, found in Section 5.4. Only four of Wardlow's sources were checked for their lensing nature, which could indicate that their low lensing fraction is caused by small-number statistics. We can also think of two physical reasons for the low lensing fractions, namely the absence of a redshift selection and the actual decrease in the lensed fraction at lower flux densities. Redshift selection lifts the probability of lensing, by ensuring the sources are drawn from the redshift space most lensed sources are in (Strandet et al. 2016). Similarly, at lower flux densities, the fraction of lensed sources decreases, as can be seen in Fig. 10.

The SPT also seems to probe similar populations to the HerBS sources, further increasing our suspicion of a high lensing fraction in our sample. A slightly less strong agreement with the ALESS sample was found, which probes deeper on a smaller part of the sky. Interestingly, Strandet et al. (2016) report a disagreement of around 2.4 standard deviations between the SPT and ALESS sample. The HerBS sample likeness to the SPT sample is larger, suggesting this sample is more similar than to the deeper ALESS sample, especially as Strandet et al. (2016) found those two samples to be different. This

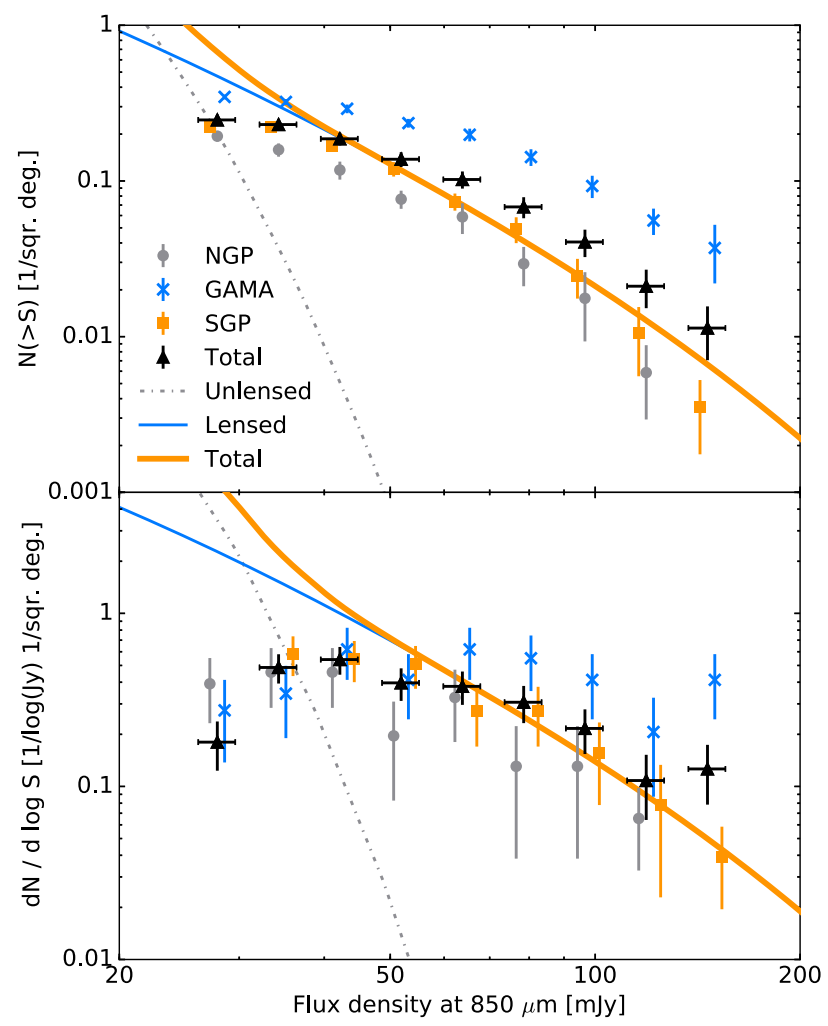

Figure 10. The top panel shows the cumulative number counts and the bottom panel shows the differential number counts of our HerBS sample, compared to the predictions of the model of Cai et al. (2013) for unlensed (dashed grey line) and lensed (solid blue line) galaxies.

is further proven by the small lensing fraction in the ALESS sample, compared to the sizeable lensing fraction in the SPT sample, and the lensing fraction we find in Section 5.4. However, Hodge et al. (2013) and Karim et al. (2013)'s studies of the ALESS sample did suggest a source confusion fraction on the order of 50 per cent of their sample. Even though our samples are not completely similar, this high blending percentage might indicate that our method of estimating the effects of source confusion with the JCMT's beam is incomplete. The low agreement to Ivison's sample suggests that their selection of unlensed SMGs was effective, and it indicates they might select different galaxies than our sample.

\subsection{Lensing fraction}

The SCUBA-2 observations do not resolve lensing directly, as the beam size (13 arcsec) is much larger than the typical Einstein rings caused by galaxy-galaxy lensing ( $\sim 1$ arcsec; Bussmann et al. 2013; 
ALMA Partnership 2015). However, we can estimate the lensing fraction of our sample when we compare the distribution of flux densities of our sources to the predictions of galaxy evolution models that include gravitational lensing.

Here we use the hybrid model by Cai et al. (2013) with a cut-off lensing magnification factor of $\mu=30$. The hybrid model is based on a parametric backward model for redshifts lower than 1.5 , whilst it calculates galaxy evolution for redshifts greater than 1.0 using physical models for the evolution of protospheroidal galaxies and their associated AGN. The model matches these two approaches to each other in the region between redshift 1.0 and 1.5. We assume all unlensed sources are high-redshift, protospheroidal galaxies. We did not observe all of the sample at $850 \mu \mathrm{m}$, so we expect that our observed number counts are a lower limit.

Fig. 10 shows a comparison of our number counts at $850 \mu \mathrm{m}$ with the predictions of the model of Cai et al. (2013). We have plotted the number counts for each of our fields, by summing the number of sources brighter than a given flux, and dividing by the corresponding area of the field, see Table 1. We estimate the error on the counts as the square root of the number of sources in each bin. A comparison of our counts with the predicted counts of the unlensed sources (grey dashed line) immediately suggests most of our sources are lensed. We can quantify this as follows.

At the low fluxes, the data deviate from the model, because of the incompleteness of the HerBS sample at fluxes lower than $\sim 50 \mathrm{mJy}$. There are more sources than the model predicts at high fluxes, the significance of which is difficult to pin down due to the small number of sources. It is possible our sources have overestimated $850 \mu \mathrm{m}$ fluxes, possibly due to source confusion. However, it is important to realize that the model of Cai et al. (2013) is based on fitted luminosity functions. The high flux end of the luminosity function requires large area surveys to be accurately fitted. As our sample is extracted from the largest area Herschel survey, the model is thus comparably uncertain as our data.

We calculate the total number of lensed sources,

$N_{\text {lens }}\left(>S_{\nu}\right)=\sum_{i}^{N_{\mathrm{gal}}\left(>S_{v}\right)} p_{\text {lens }}\left(S_{v, i}\right)$.

We sum the lensing probability, $p_{\text {lens }}\left(S_{\nu, i}\right)$, over all galaxies brighter than the flux cut-off, $N_{\text {gal }}\left(>S_{v}\right)$. We calculate the probability, $p_{\text {lens }}\left(S_{v, i}\right)$, from the relative proportions of the differential number counts predicted for lensed and unlensed galaxies,

$p_{\text {lens }}\left(S_{v, i}\right)=\left[\frac{\mathrm{d} N_{\text {lens }}}{\mathrm{d} S_{v}} /\left(\frac{\mathrm{d} N_{\text {proto }}}{\mathrm{d} S_{v}}+\frac{\mathrm{d} N_{\text {lens }}}{\mathrm{d} S_{v}}\right)\right]_{S_{v, i}}$.

The $N_{\text {lens }}$ term refers to the lensed sources, and the $N_{\text {proto }}$ term refers to the unlensed protospheroidal galaxies. We evaluate the probability at the flux density of the source, $S_{v, i}$. Using the bottom panel of Fig. 10, $p_{\text {lens }}$ can be thought of as the fraction lenses (thin blue line) over the total sources (thick orange line).

We iterate this procedure a 1000 times, varying the $850 \mu \mathrm{m}$ flux with a Gaussian distribution with a width of the measurement uncertainty. Table 7 shows the predicted number of lensed sources (equation 7) and the observed number of sources for all SCUBA-2 detected HerBS sources. All of the errors are the standard deviations. Even for sources at $S_{850 \mu \mathrm{m}}>30 \mathrm{mJy}$, the predicted lensing fraction is $\sim 92$ percent, increasing to nearly all sources with $S_{850 \mu \mathrm{m}}>40 \mathrm{mJy}$.

We rerun the same procedure on the $500 \mu \mathrm{m}$ SPIRE fluxes, which shows that out of all 209 HerBS sources, we expect $158.1 \pm 1.7$ lensed sources, giving a total lensing fraction of $75.6 \pm 0.8$ per cent.
Table 7. Predicted lenses in the HerBS sample.

\begin{tabular}{lrrr}
\hline$S_{850 \mu \mathrm{m}}(\mathrm{mJy})$ & $N\left(>\mathrm{S}_{850 \mu \mathrm{m}}\right)$ & Lenses & Percentage \\
\hline All & $152.0 \pm 0.0$ & $128.4 \pm 2.1$ & $84.5 \pm 1.4$ \\
30 & $133.8 \pm 3.4$ & $123.3 \pm 2.9$ & $92.2 \pm 0.9$ \\
40 & $107.6 \pm 3.9$ & $105.2 \pm 3.7$ & $97.8 \pm 0.3$ \\
50 & $80.8 \pm 3.6$ & $80.5 \pm 3.6$ & $99.6 \pm 0.1$ \\
60 & $60.0 \pm 3.2$ & $59.9 \pm 3.2$ & $99.9 \pm 0.0$ \\
70 & $44.2 \pm 2.9$ & $44.2 \pm 2.9$ & $100.0 \pm 0.0$ \\
80 & $32.4 \pm 2.4$ & $32.4 \pm 2.4$ & $100.0 \pm 0.0$ \\
90 & $23.7 \pm 2.0$ & $23.7 \pm 2.0$ & $100.0 \pm 0.0$ \\
100 & $17.4 \pm 1.7$ & $17.4 \pm 1.7$ & $100.0 \pm 0.0$ \\
120 & $9.5 \pm 1.3$ & $9.5 \pm 1.3$ & $100.0 \pm 0.0$ \\
\hline
\end{tabular}

This suggests that we are missing $29.7 \pm 1.6$ lensed sources with our SCUBA-2 observations.

Finally, we note that our counts in the GAMA fields are systematically higher than those in the other H-ATLAS fields, a point also noticed by Negrello et al. (2017). Using a similar method for the KS test as described in Section 5.3, we calculate the probability for the GAMA and non-GAMA sources, and find a disagreement of $0.61 \pm 0.47$ standard deviations. This suggests the sources themselves do not differ significantly between the GAMA and the NGP+SGP fields.

\section{CONCLUSIONS}

The HerBS catalogue consists of the brightest, high-redshift sources in the H-ATLAS survey, selected with $S_{500 \mu \mathrm{m}}>80 \mathrm{mJy}$ and $z_{\text {phot }}>2$. Initially, we selected 223 sources. SCUBA-2 observations of 203 of these sources allowed us to remove 14 blazars from the HerBS sample, leaving 20 HerBS sources unobserved. 152 out of the 189 confirmed high-redshift galaxies were detected at more than $3 \sigma$, within 10 arcsec of the SPIRE position. Currently, our HerBS sample consists of 209 galaxies.

While recent studies like Scudder et al. (2016) suggest a significant effect of source confusion in Herschel observations, none of our sources feature spatially extended emission with $>3 \sigma$. While some sources could be confused on a scale not probed by the SCUBA-2 observations, the lack of any signs at the detectable scales gives us little evidence of source confusion significantly affecting the purity of our sample. A reason for this could be due to our high lensing fraction, especially those caused by galaxy-galaxy lensing systems, whose influence is on a smaller angular scale than the less common galaxy-cluster lensing event.

We fitted a two-temperature blackbody as a template to the subset of 22 HerBS sources with spectroscopically determined redshifts, as well as to subsamples where we divided our sources in redshift or luminosity. We find a cold- and hot-dust temperature of $21.29_{-1.66}^{+1.35}$ and $45.80_{-3.48}^{+2.88} \mathrm{~K}$, a cold-to-hot dust mass ratio of $26.62_{-6.74}^{+5.61}$ and a $\beta$ of $1.83_{-0.28}^{+0.14}$. Overall, the fitted parameters are similar to previous work from Pearson et al. (2013), and they agree broadly with the previous work from Dunne \& Eales (2001) and Clements et al. (2010). We do not find evidence of any cold gas with temperatures below $20 \mathrm{~K}$, as was found in Planck Collaboration XVI (2011).

We find a high $\chi^{2}$ for the template, implying that the SEDs of the high-redshift population are diverse and cannot be represented by a single template. We showed that our improved template, which incorporates the SCUBA-2 flux densities, does not give a more accurate redshift estimates, which can also be explained by the diversity of the population. 
Our sample has a similar redshift distribution as other samples selected at $500 \mu \mathrm{m}$, when we take the photometric redshift cut-off into account. KS tests indicate that we probe a similar sample of galaxies as the SPT sample.

We calculated the number counts of the $850 \mu \mathrm{m}$ observations of our sources, and compared them to a galaxy population model by Cai et al. (2013). From this comparison we predict that $128.4 \pm 2.1$ out of the 152 SCUBA-2 detected, high-redshift galaxies are strongly lensed. A model based around the $500 \mu \mathrm{m}$ flux suggests a total of $158.1 \pm 1.7$ of the 209 HerBS sources to be strongly lensed. We report finding more lensed galaxies in the GAMA equatorial fields, when compared to the galaxy population model of Cai et al. (2013), and the other fields (SGP+NGP).

\section{ACKNOWLEDGEMENTS}

We would like to thank our JCMT operator, James Hoge, for his help at the full-service James Clerck Maxwell Telescope. TJLCB, MWLS and SAE have received funding from the European Union Seventh Framework Programme ([FP7/2007-2013] [FP\&/20072011]) under grant agreement no. 607254. The Herschel-ATLAS is a project with Herschel, which is an ESA space observatory with science instruments provided by European-led Principal Investigator consortia and with important participation from NASA. The Herschel-ATLAS website is http://www.h-atlas.org. RJI acknowledges support from ERC in the form of the Advanced Investigator Programme, 321302, COSMICISM. LD and SM acknowledge support from ERC in the form of the Advanced Investigator Programme, 321302, COSMICISM, and the Consolidator Grant COSMICDUST (ERC-2014-CoG-647939, PI: H. L. Gomez). MJM acknowledges the support of the National Science Centre, Poland, through the POLONEZ grant 2015/19/P/ST9/04010. This project has received funding from the European Union's Horizon 2020 research and innovation programme under the Marie Skłodowska-Curie grant agreement no. 665778. MN acknowledges financial support from the European Union's Horizon 2020 research and innovation programme under the Marie Skłodowska-Curie grant agreement no. 707601. HD acknowledges financial support from the Spanish Ministry of Economy and Competitiveness (MINECO) under the 2014 Ramón y Cajal program MINECO RYC-2014-15686. This research has made use of the NASA/IPAC Extragalactic Database (NED) which is operated by the Jet Propulsion Laboratory, California Institute of Technology, under contract with the National Aeronautics and Space Administration. The authors thank the anonymous referee for comments and suggestions.

\section{REFERENCES}

ALMA Partnership, 2015, ApJ, 808, L4

Avni Y., 1976, ApJ, 210, 642

Bethermin M., De Breuck C., Sargent M., Daddi E., 2015, A\&A, 576, L9

Blain A. W., Longair M. S., 1993, MNRAS, 264, 509

Blain A., Perrotta F., González J. G.-N., Silva L., De Zotti G., Granato G.

L., Baccigalupi C., Danese L., 1999, ApJ, 512, L87

Blain A. W., Smail I., Ivison R. J., Kneib J. P., Frayer D. T., 2002, Phys. Rep., 369, 111

Borys C. et al., 2006, ApJ, 636, 134

Bussmann R. S. et al., 2012, ApJ, 756, 134

Bussmann R. S. et al., 2013, ApJ, 779, 25

Cai Z.-Y. et al., 2013, ApJ, 768, 21

Casey C. M., Narayanan D., Cooray A., 2014, Phys. Rep., 541, 45

Chapin E. L., Berry D. S., Gibb A. G., Jenness T., Scott D., Tilanus R. P. J., Economou F., Holland W. S., 2013, MNRAS, 430, 2545
Chapman S. C., Perrotta F., González J. G.-N., Silva L., De Zotti G., Granato G. L., Baccigalupi C., Danese L., 2005, ApJ, 622, 772

Chen C.-C., Cowie L. L., Barger A. J., Casey C. M., Lee N., Sanders D. B., Wang W.-H., Williams J. P., 2013, ApJ, 776, 131

Chapin E., Dempsey J., Jenness T., Scott D., Thomas H., Tilanus R., 2010, The SCUBA-2 SRO Data Reduction Cookbook, Starlink Cookbook 19

Chen C.-C. et al., 2016, ApJ, 820, 82

Clements D. L., Dunne L., Eales S., 2010, MNRAS, 403, 274

Conley A. et al., 2011, ApJ, 732, L35

Cox P. et al., 2011, ApJ, 740, 63

Dempsey J. T. et al., 2013, MNRAS, 430, 2534

Driver S. P. et al., 2011, MNRAS, 413, 971

Dunne L., Eales S. A., 2001, MNRAS, 327, 697

Dye S. et al., 2015, MNRAS, 452, 2258

Eales S. A., 2015, MNRAS, 446, 3224

Eales S. et al., 2010, PASP, 122, 499

Frayer D. T. et al., 2011, ApJ, 726, L22

Fu H. et al., 2012, ApJ, 753, 134

George R. D. et al., 2013, MNRAS, 436, L99

González-Nuevo J. et al., 2012, ApJ, 749, 65

Griffin M. J. et al., 2010, A\&A, 518, L3

Harris A. I. et al., 2012, ApJ, 752, 152

Hatsukade B., Tamura Y., Iono D., Matsuda Y., Hayashi M., Oguri M., 2015, PASJ, 67, 93

Hayward C. C., Behroozi P. S., Somerville R. S., Primack J. R., Moreno J., Wechsler R. H., 2013, MNRAS, 434, 2572

Hezaveh Y. D. et al., 2016a, ApJ, 823, 37

Hezaveh Y. D., Perrotta F., González J. G.-N., Silva L., De Zotti G., Granato G. L., Baccigalupi C., Danese L., 2016b, J. Cosmol. Astropart. Phys., 11,048

Hodge J. A. et al., 2013, ApJ, 768, 91

Holland W. S. et al., 2013, MNRAS, 430, 2513

Hughes D. H. et al., 1998, Nature, 394, 241

Ikarashi S. et al., 2011, MNRAS, 415, 3081

Ivison R. J. et al., 2010, A\&A, 518, L35

Ivison R. J. et al., 2013, ApJ, 772, 137

Ivison R. J. et al., 2016, ApJ, 832, 78

Karim A. et al., 2013, MNRAS, 432, 2

Koprowski M. P., Dunlop J. S., Michałowski M. J., Cirasuolo M., Bowler R. A. A., 2014, MNRAS, 444, 117

Liske J. et al., 2015, MNRAS, 452, 2087

López-Caniego M. et al., 2013, MNRAS, 430, 1566

Lupu R. E. et al., 2012, ApJ, 757, 135

Madau P., Dickinson M., 2014, ARA\&A, 52, 415

Nayyeri H., Perrotta F., González J. G.-N., Silva L., De Zotti G., Granato G. L., Baccigalupi C., Danese L., 2016, ApJ, 823, 17

Negrello M., Perrotta F., González J. G.-N., Silva L., De Zotti G., Granato G. L., Baccigalupi C., Danese L., 2007, MNRAS, 377, 1557

Negrello M. et al., 2010, Science, 330, 800

Negrello M. et al., 2017, MNRAS, 465, 3558

Oguri M., Perrotta F., González J. G.-N., Silva L., De Zotti G., Granato G. L., Baccigalupi C., Danese L., 2012, AJ, 143, 120

Pearson E. A. et al., 2013, MNRAS, 435, 2753

Pilbratt G. L. et al., 2010, A\&A, 518, L1

Planck Collaboration XVI, 2011, A\&A, 536, A16

Planck Collaboration XIII, 2016, A\&A, 594, A13

Poglitsch A. et al., 2010, A\&A, 518, L2

Pope A. et al., 2008, ApJ, 689, 127

Rybak M., McKean J. P., Vegetti S., Andreani P., White S. D. M., 2015, MNRAS, 451, L40

Sawicki M., 2012, PASP, 124, 1208

Schaye J. et al., 2015, MNRAS, 446, 521

Scudder J. M., Oliver S., Hurley P. D., Griffin M., Sargent M. T., Scott D., Wang L., Wardlow J. L., 2016, MNRAS, 460, 1119

Simpson J. M., Perrotta F., González J. G.-N., Silva L., De Zotti G., Granato G. L., Baccigalupi C., Danese L., 2014, ApJ, 788, 125 
Smail I., Perrotta F., González J. G.-N., Silva L., De Zotti G., Granato G. L., Baccigalupi C., Danese L., 2002, MNRAS, 331, 495

Springel V. et al., 2005, Nature, 435, 629

Strandet M. L. et al., 2016, ApJ, 822, 80

Swinbank A. M. et al., 2010, Nature, 464, 733

Swinbank A. M. et al., 2014, MNRAS, 438, 1267

Swinbank A. M. et al., 2015, ApJ, 806, L17

Tamura Y., Oguri M., Iono D., Hatsukade B., Matsuda Y., Hayashi M., 2015, PASJ, 67, 72

Thomson A. P. et al., 2017, ApJ, 838, 119

Treu T., 2010, ARA\&A, 48, 87

Valiante E. et al., 2016, MNRAS, 462, 3146

Vegetti S., Lagattuta D. J., McKean J. P., Auger M. W., Fassnacht C. D., Koopmans L. V. E., 2012, Nature, 481, 341

Wardlow J. L. et al., 2011, MNRAS, 415, 1479

Wardlow J. L., Perrotta F., González J. G.-N., Silva L., De Zotti G., Granato G. L., Baccigalupi C., Danese L., 2013, ApJ, 762, 59

Weiß A. et al., 2009, ApJ, 707, 1201
Weiß A. et al., 2013, ApJ, 767, 88

Zavala J. A. et al., 2015, MNRAS, 452, 1140

\section{SUPPORTING INFORMATION}

Supplementary data are available at MNRAS online.

Please note: Oxford University Press is not responsible for the content or functionality of any supporting materials supplied by the authors. Any queries (other than missing material) should be directed to the corresponding author for the article.

\author{
APPENDIX A: HerBS CATALOGUE AND \\ BLAZARS
}


ํ.

๑ัँ

쿠

过

品

ปี

范

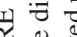

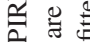

is

政

نั

ज

«

焉

نं

言 自

品

㟧.

.

政

o:

㲾

के

范

焉

画

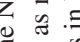

$\stackrel{0}{ \pm}$

일

节

可

솔 흘

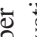

表

กิ

过安

记

赵证

苞 密

兒

ष.

雚:

을

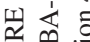

空

을

要

家

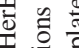

T.

要要

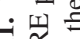

$\underset{4}{2}$

造 की

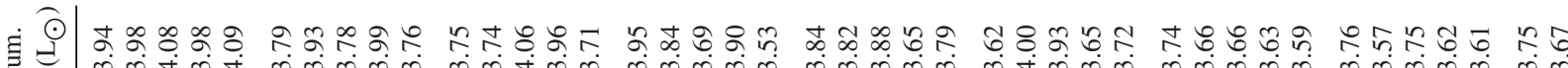

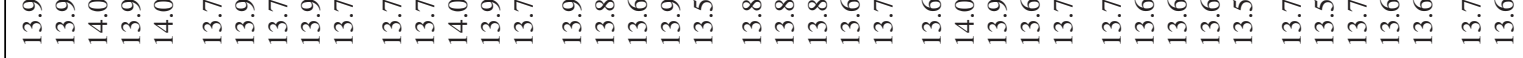

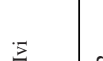

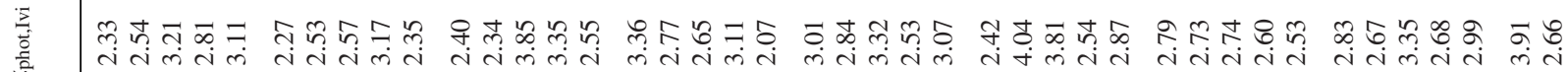

a.

高

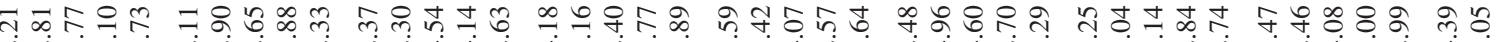

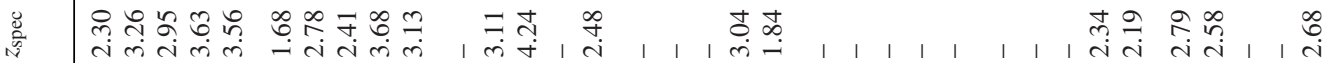

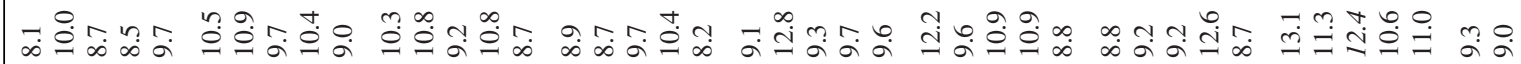
H H H H H H H H H H $\quad$ H H H H H

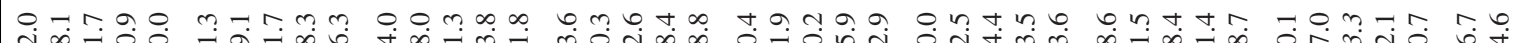

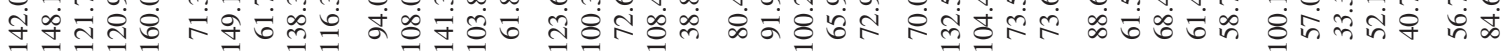

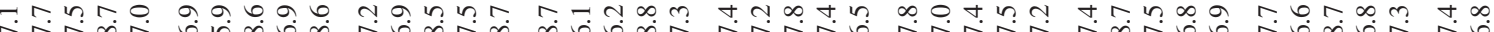

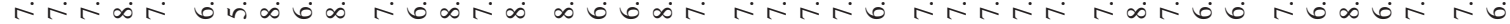

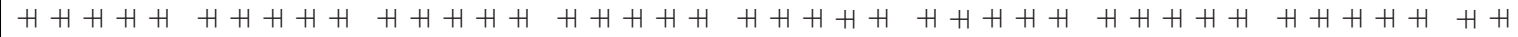

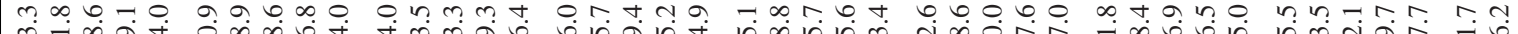

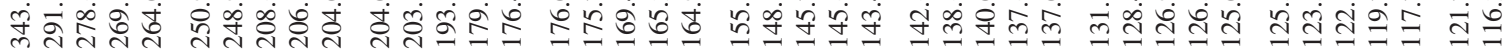

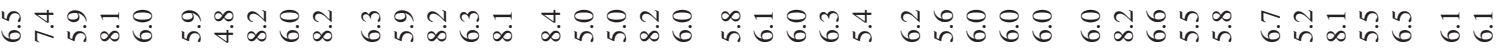

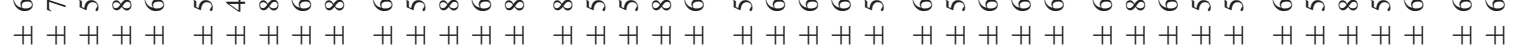
r.

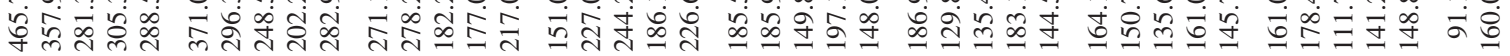

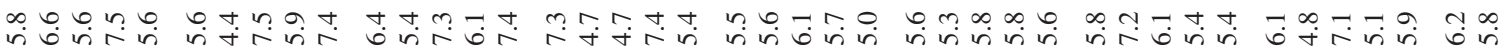

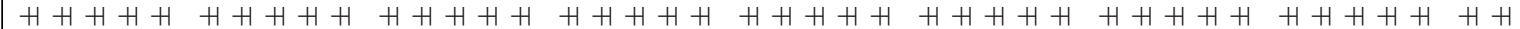

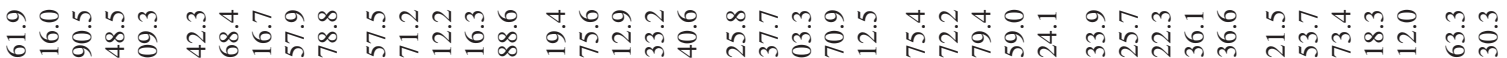

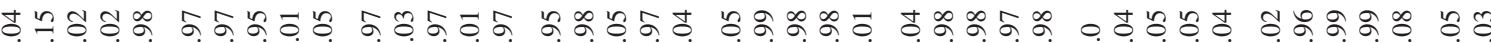

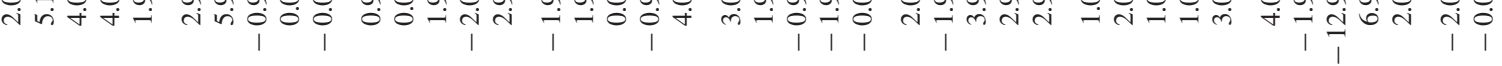

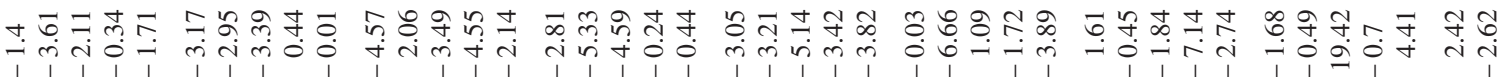

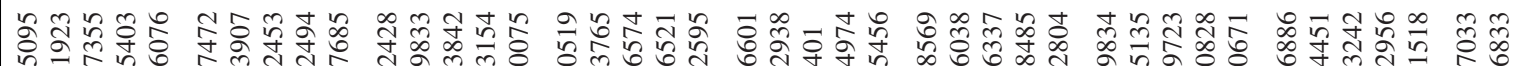

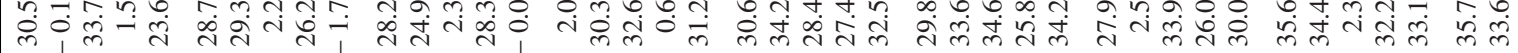

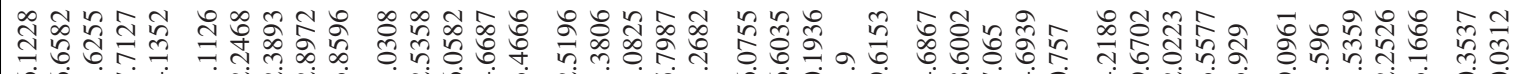

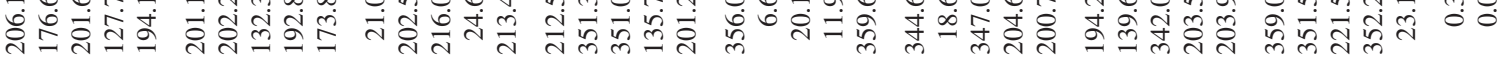

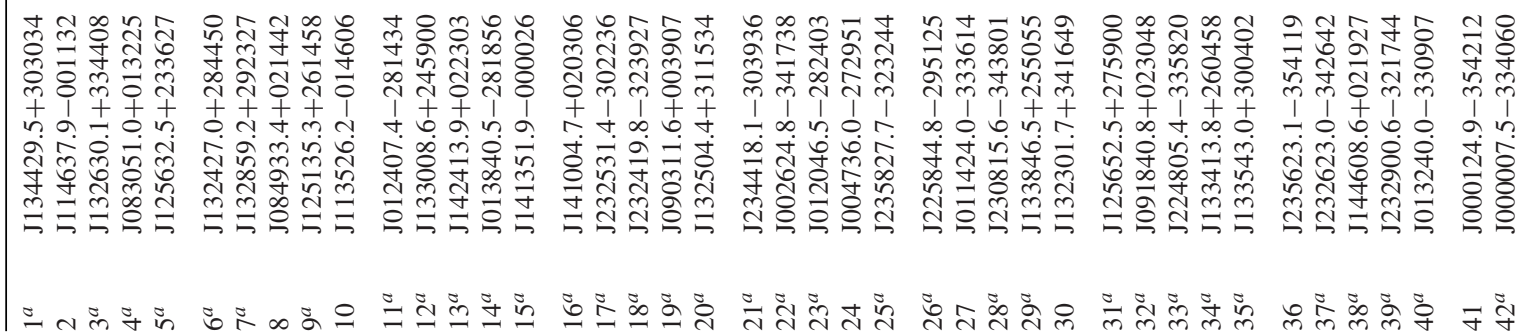




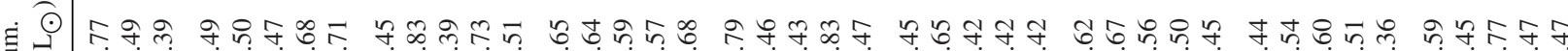

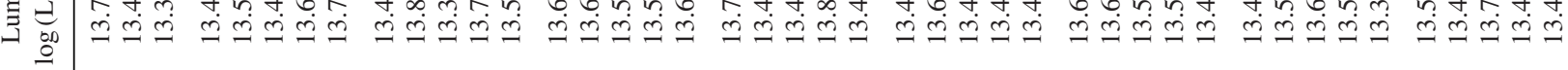

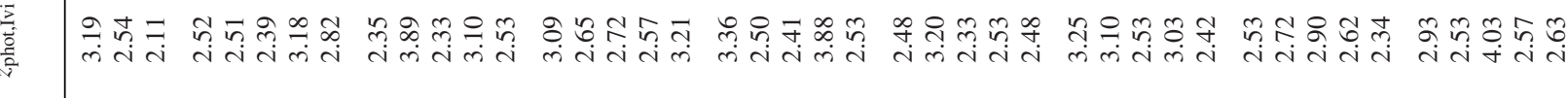
덩ำ 竞

$\stackrel{\infty}{i}$

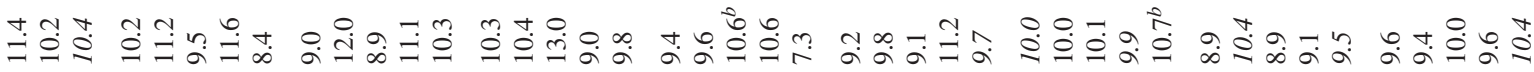

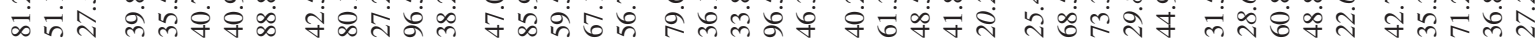

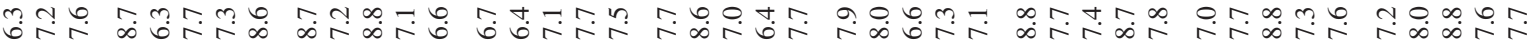
H H H H H H H H H H H H H H H H H H H H H H H H H H H H H H H H H H H H H H H H H H H

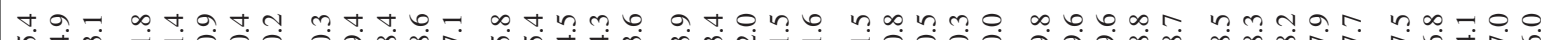

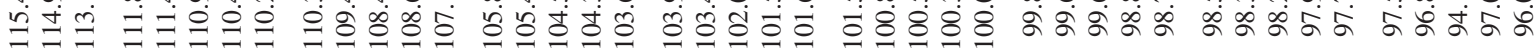

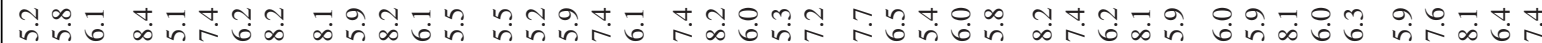
宓家

苛

级 $H+H+H+H+H+H+H+H+H+H+H+H+H+H+H+H+H+H+H+H+H+H+H+H+H+H$

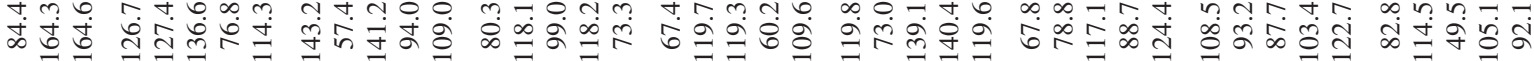

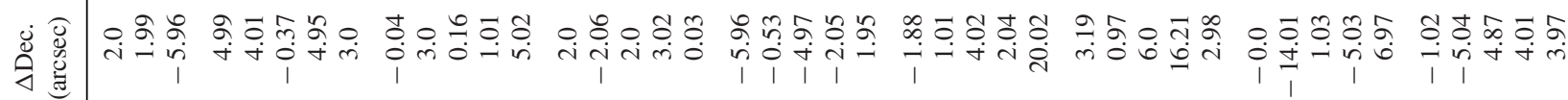

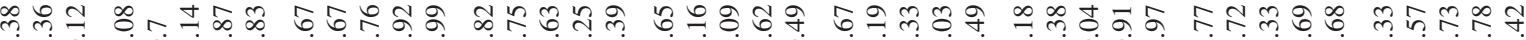
mi mili

चके m㠃

$\alpha$ १ 1 i 1 तो

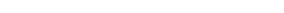

$$
\begin{array}{lll}
1 & 1 & 1
\end{array}
$$

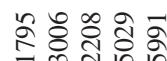

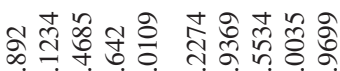

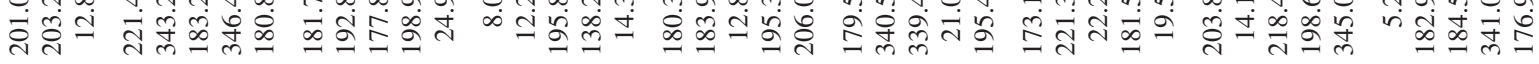

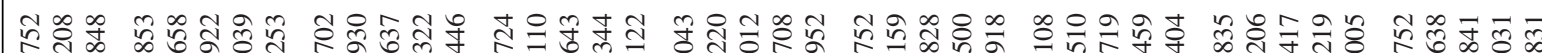

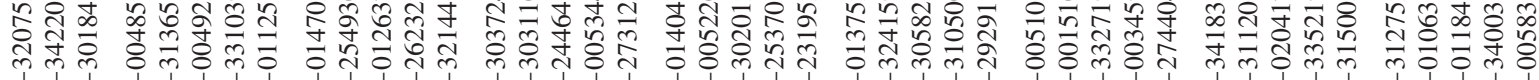

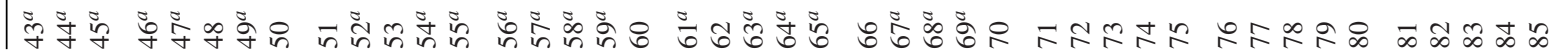




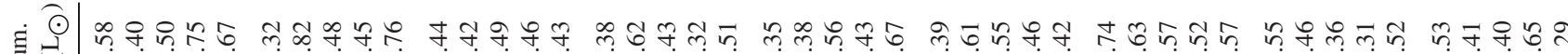
三丨

商

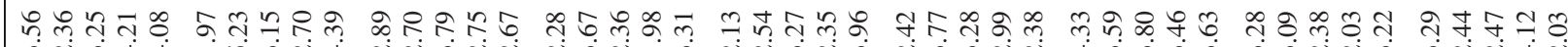

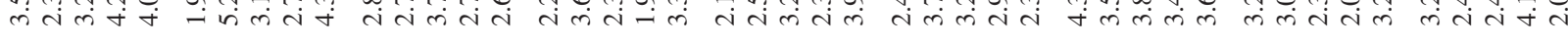

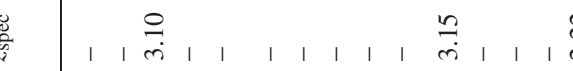

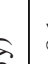

-

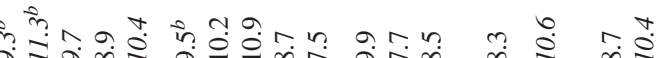

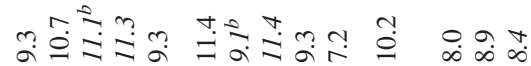

$\stackrel{\infty}{\infty}$

$\mathrm{H}+\mathrm{H}+\mathrm{H}+\mathrm{H}, \mathrm{H}+\mathrm{H}$

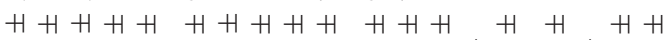

H H H H H H H H H H

$H+H$

$0 m+\infty+0, n m 1$ n

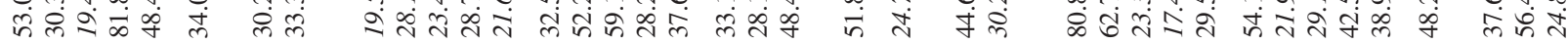

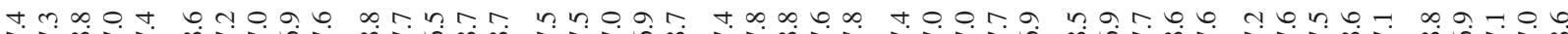

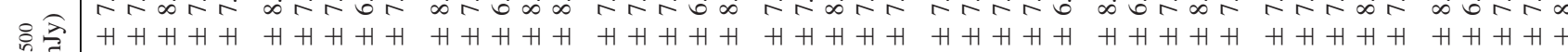

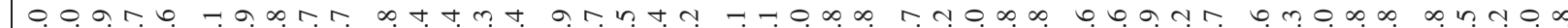

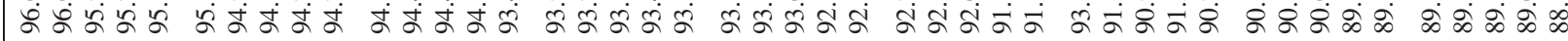

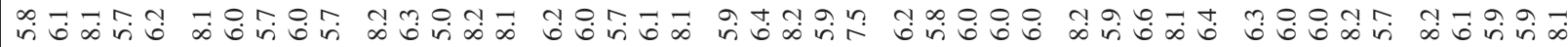
$H+H+H+H+H+H+H \quad H+H+H+H \quad H+H+H+H+H+H+H+H+H+H+H+H+H \quad H+H+H+H+H+H+H$

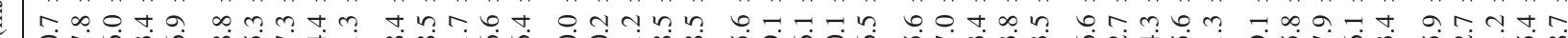
๙்

ம $H+H+H+H+H+H+H \quad H+H+H+H+H+H+H \quad H+H+H+H+H+H+H+H+H+H+H+H+H+H+H+H+H$

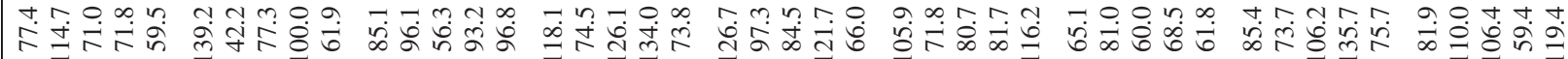

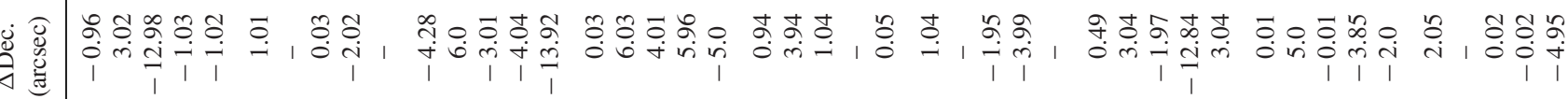

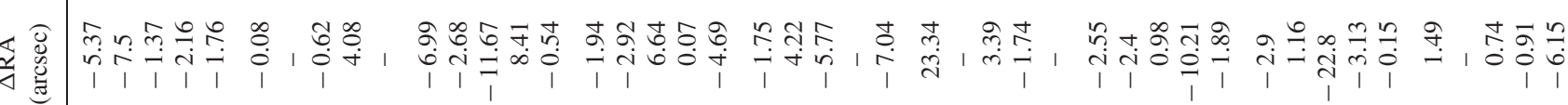

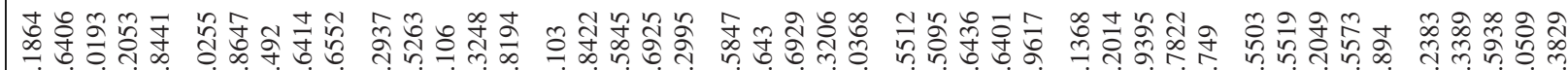

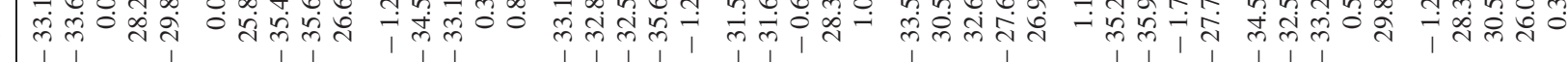

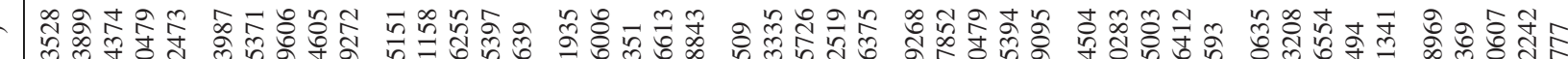
管

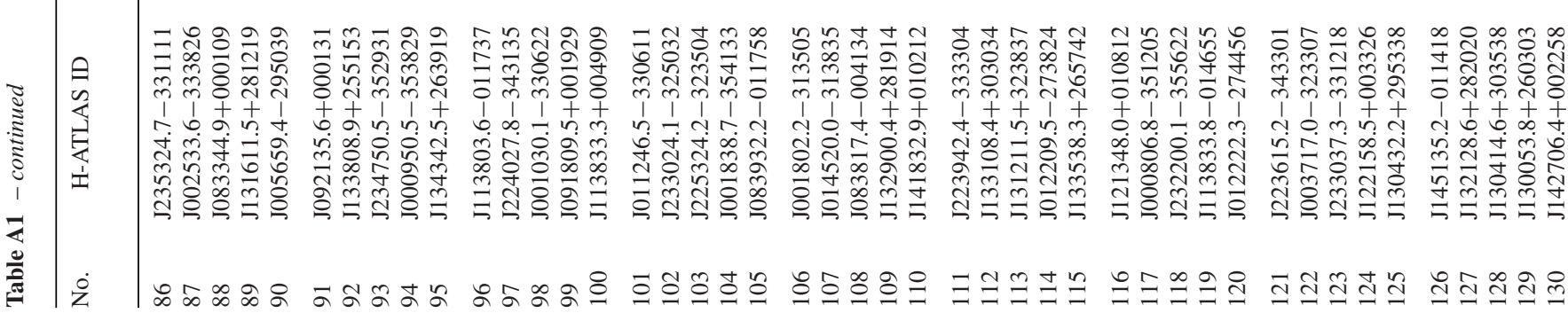




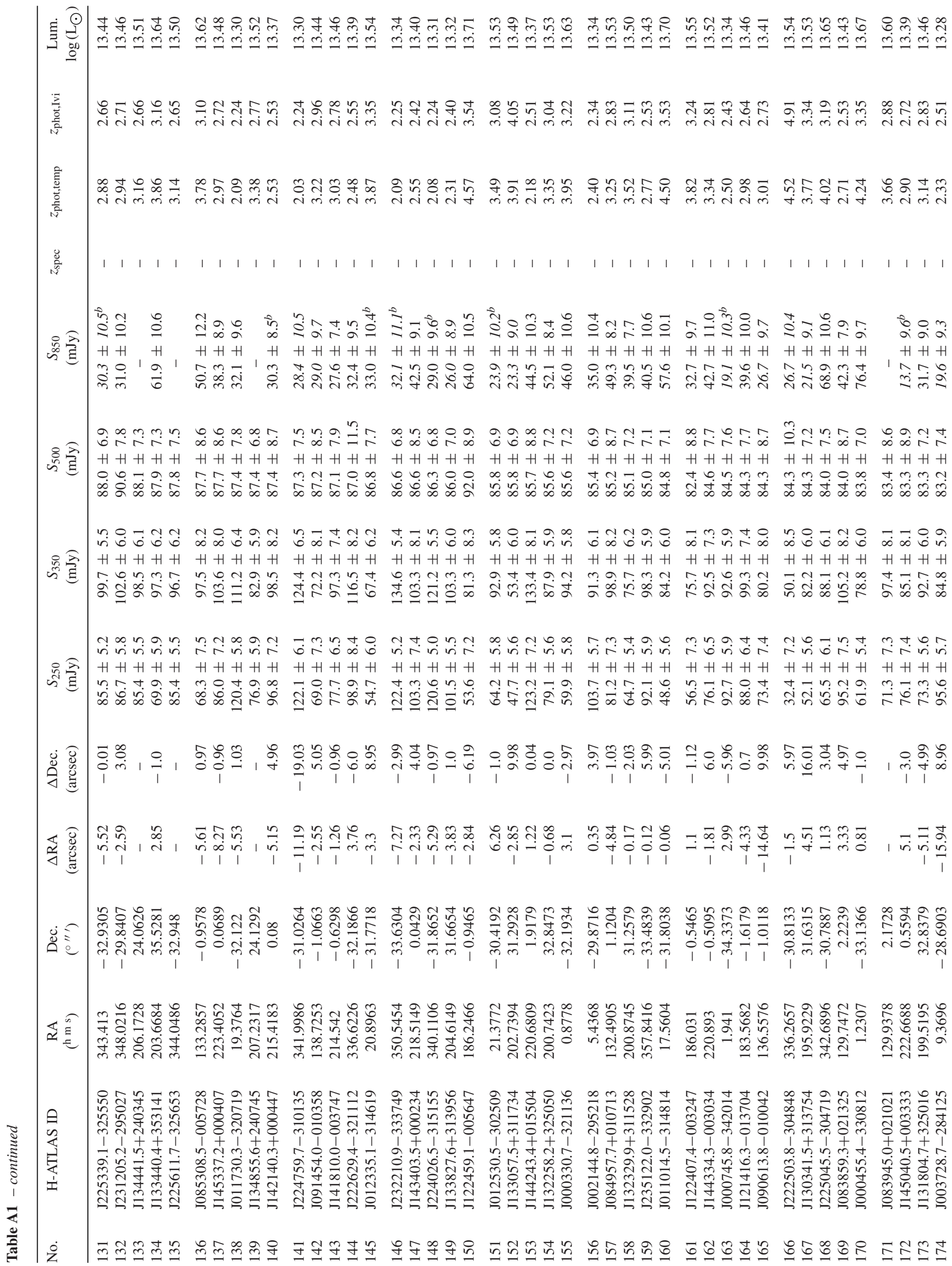




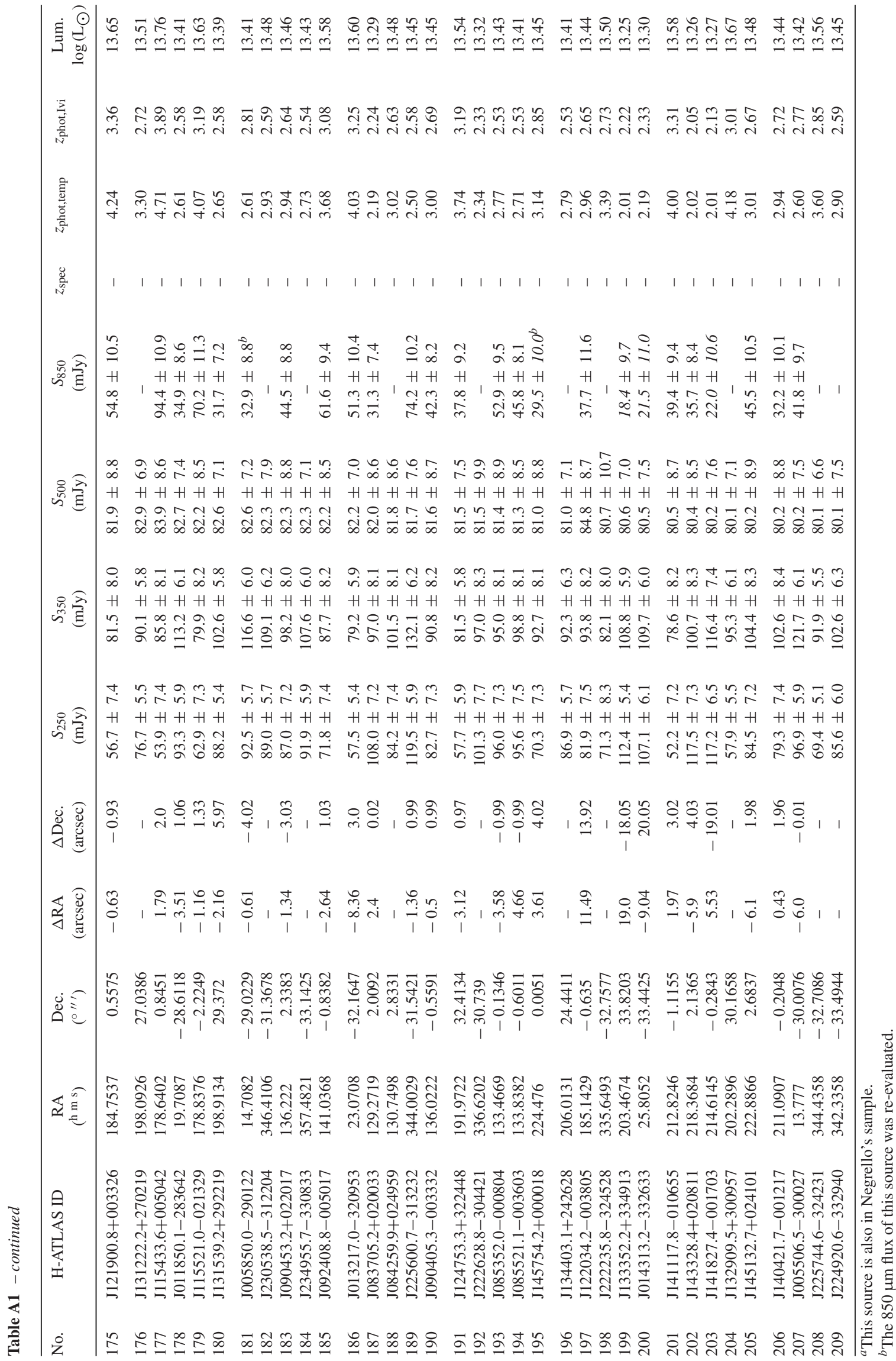




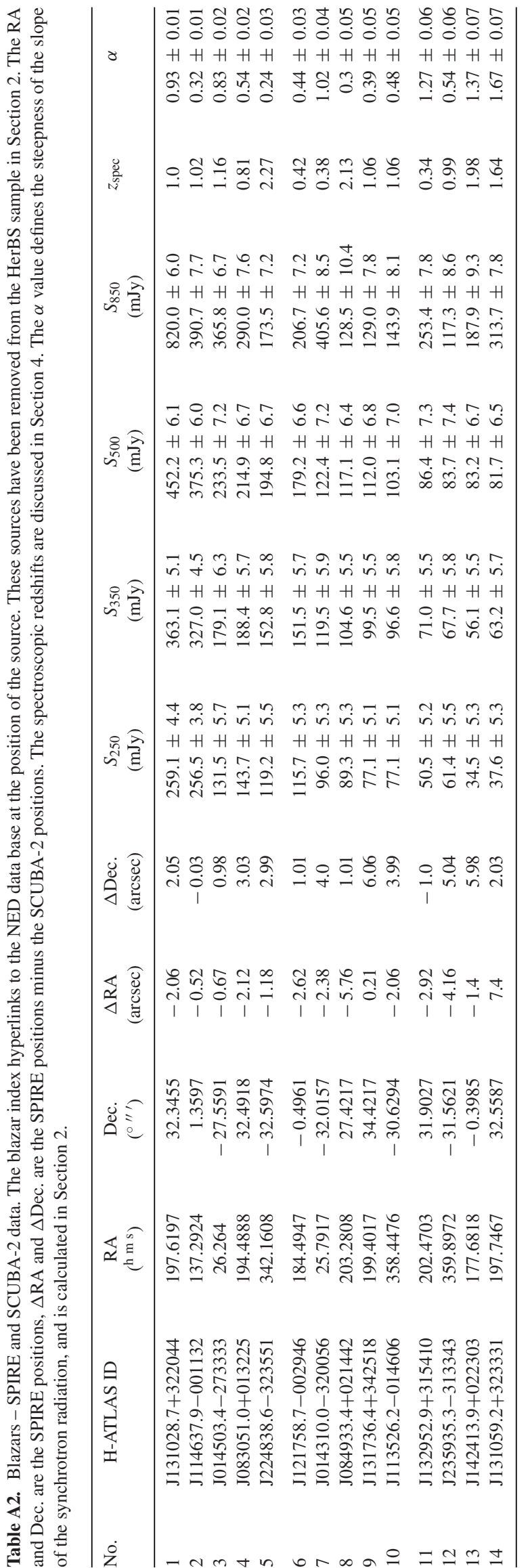




\section{APPENDIX B: CUTOUTS OF THE ENTIRE \\ HERBS SAMPLE}

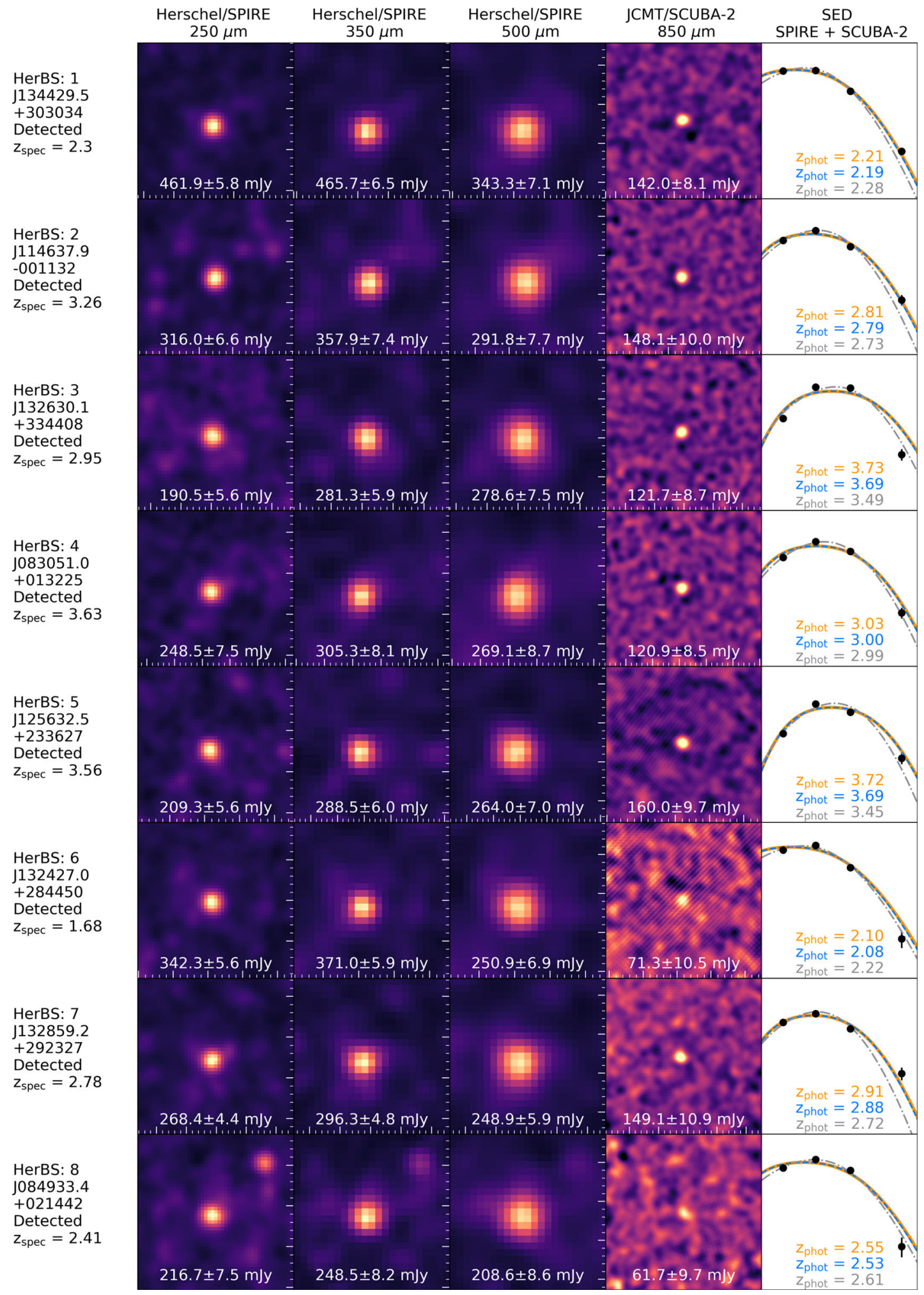

Figure B1. The cutouts of the first 8 HerBS sources. The cutouts of all HerBS sources can be found in the online version of this paper. The first three columns of cutouts of each source are the Herschel observations shown in $4 \times 4 \operatorname{arcmin}^{2}$ postage stamps. The fourth column shows the $850 \mu \mathrm{m}$ SCUBA-2 observation, where available, in a $4 \times 4 \operatorname{arcmin}^{2}$ postage stamp. All postage stamps are centred at the $250 \mu \mathrm{m}$ extraction position of the Herschel catalogue. The final frame is a fitted SED, with the best-fitting template in orange, fixed $\beta$ template in blue and Pearson's template in grey (Pearson et al. 2013). 
${ }^{1}$ School of Physics and Astronomy, Cardiff University, The Parade, Cardiff CF24 3AA, UK

${ }^{2}$ UK Astronomy Technology Centre, Royal Observatory, Blackford Hill, Edinburgh EH9 3HJ, UK

${ }^{3}$ Sterrenkundig Observatorium, Universiteit Gent, Krijgslaan 281 S9, B-9000 Gent, Belgium

${ }^{4}$ Institute for Astronomy, University of Edinburgh, Royal Observatory, Edinburgh EH9 3HJ, UK

${ }^{5}$ Astrophysics Group, Imperial College, Blackett Laboratory, Prince Consort Road, London SW7 2AZ, UK

${ }^{6}$ Institut für Astrophysik, Universität Wien, Türkenschanzstrabe 17, A-1180 Wien, Austria

${ }^{7}$ Instituto de Astrofísica de Canarias (IAC), E-38205 La Laguna, Tenerife, Spain

${ }^{8}$ Dpto. Astrofísica, Universidad de La Laguna, E-38206 La Laguna, Tenerife, Spain

${ }^{9}$ INAF - Osservatorio Astronomico di Padova, Vicolo dell'Osservatorio 5, I-35122 Padova, Italy

${ }^{10}$ School of Physics and Astronomy, University of Nottingham, University Park, Nottingham NG7 2RD, UK

${ }^{11}$ CAPES Foundation, Ministry of Education of Brazil, 70040-020 Brasília, DF, Brazil
${ }^{12}$ European Southern Observatory, Karl-Schwarzschild-Strasse 2, D-85748 Garching bei München, Germany

${ }^{13}$ Department of Physical Science, The Open University, Milton Keynes $M K 7$ 6AA, UK

${ }^{14}$ Astronomical Observatory Institute, Faculty of Physics, Adam Mickiewicz University, ul. Stoneczna 36, PL-60-286 Poznań, Poland

${ }^{15}$ UPMC Univ. Paris 06, UMR7095, Institut d'Astrophysique de Paris, F75014 Paris, France

${ }^{16}$ CNRS, UMR7095, Institut d'Astrophysique de Paris, F-75014 Paris, France

${ }^{17}$ Centre for Extragalactic Astronomy, Department of Physics, Durham University, South Road, Durham DH1 3LE, UK

${ }^{18}$ Leiden Observatory, Leiden University, PO Box 9513, NL-2300 RA Leiden, the Netherlands

${ }^{19}$ Purple Mountain Observatory/Key Lab of Radio Astronomy, Chinese Academy of Sciences, Nanjing 210008, China

${ }^{20}$ Institut d'Astrophysique Spatiale, CNRS, Univ. Paris-Sud, Université Paris-Saclay, Bât. 121, F-91405 Orsay Cedex, France

${ }^{21}$ Graduate University of the Chinese Academy of Sciences, 19A Yuquan Road, Shijingshan District, Beijing 10049, China

This paper has been typeset from a $\mathrm{T}_{\mathrm{E}} \mathrm{X} / \mathrm{LT} \mathrm{E} \mathrm{X}$ file prepared by the author. 